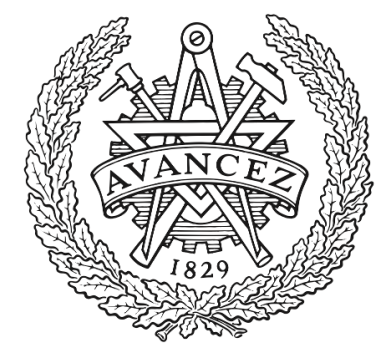

CHALMERS

UNIVERSITY OF TECHNOLOGY

\title{
Multiwavelength Observations of the RV Tauri Variable System U Monocerotis: Long-term Variability Phenomena That Can Be Explained by
}

Downloaded from: https://research.chalmers.se, 2023-04-26 10:20 UTC

Citation for the original published paper (version of record):

Vega, L., Stassun, K., Montez et al (2021). Multiwavelength Observations of the RV Tauri Variable System U Monocerotis: Long-term

Variability Phenomena That Can Be Explained by Binary Interactions with a Circumbinary Disk. Astrophysical Journal, 909(2). http://dx.doi.org/10.3847/1538-4357/abe302

N.B. When citing this work, cite the original published paper. 


\title{
Multiwavelength Observations of the RV Tauri Variable System U Monocerotis: Long- term Variability Phenomena That Can Be Explained by Binary Interactions with a Circumbinary Disk
}

\author{
Laura D. Vega ${ }^{1,2,10,11}$ (), Keivan G. Stassun ${ }^{1}(\mathbb{0})$, Rodolfo Montez, Jr. ${ }^{3}$ (1) , Tomasz Kamiński ${ }^{4}$ (1), Laurence $\operatorname{Sabin}^{5}$ (1), \\ Eric M. Schlegel ${ }^{6}$ (10), Wouter H. T. Vlemmings ${ }^{7}$ (1) , Joel H. Kastner ${ }^{8}$ (1), Sofia Ramstedt ${ }^{9}$ (1), and Patricia T. Boyd ${ }^{2}$ (1) \\ ${ }^{1}$ Department of Physics \& Astronomy, Vanderbilt University, Nashville, TN 37235, USA \\ ${ }^{2}$ Astrophysics Science Division, NASA Goddard Space Flight Center, Greenbelt, MD 20771, USA \\ ${ }^{3}$ Center for Astrophysics | Harvard \& Smithsonian, Cambridge, MA 02138, USA \\ ${ }_{5}^{4}$ Nicolaus Copernicus Astronomical Center of the Polish Academy of Sciences, Toruń, Poland \\ ${ }^{5}$ Instituto de Astronomía, Universidad Nacional Autónoma de México, Ensenada, B.C., México \\ ${ }^{6}$ Department of Physics \& Astronomy, The University of Texas at San Antonio, San Antonio, TX 78249, USA \\ ${ }^{7}$ Department of Space, Earth and Environment, Chalmers University of Technology, Onsala Space Observatory, Onsala, Sweden \\ ${ }^{8}$ School of Physics and Astronomy, Rochester Institute of Technology, Rochester, NY 14623, USA \\ ${ }^{9}$ Department of Physics and Astronomy, Uppsala University, Uppsala, Sweden \\ Received 2020 August 3; revised 2021 January 29; accepted 2021 February 2; published 2021 March 12
}

\begin{abstract}
We present an X-ray through submillimeter observations of the classical RV Tauri (RVb-type) variable U Mon, a post-asymptotic giant branch (AGB) binary with a circumbinary disk (CBD). Our SMA observations indicate a CBD diameter of $\lesssim 550$ au. Our XMM-Newton observations make U Mon the first RV Tauri variable detected in $\mathrm{X}$-rays. The X-ray emission is characteristic of a hot plasma $(\sim 10 \mathrm{MK})$, with $L_{\mathrm{X}}=5 \times 10^{30} \mathrm{erg} \mathrm{s}^{-1}$, and we consider its possible origin from U Mon, its companion, and/or binary system interactions. Combining DASCH and AAVSO data, we extend the time-series photometric baseline back to the late 1880s and find evidence that U Mon has secular changes that appear to recur on a timescale of $\sim 60 \mathrm{yr}$, possibly caused by a feature in the CBD. From literature radial velocities we find that the binary companion is a $\sim 2 M_{\odot}$ A-type main-sequence star. The orientation of the binary's orbit lies along our line of sight $\left(\omega=95^{\circ}\right)$, such that apastron corresponds to photometric $\mathrm{RVb}$ minima, consistent with the post-AGB star becoming obscured by the near side of the CBD. In addition, we find the size of the inner-CBD hole ( 4.5-9 au) to be comparable to the binary separation, implying that one or both stars may interact with the CBD at apastron. The obscuration of the post-AGB star implicates the companion as the likely source of the enhanced $\mathrm{H} \alpha$ observed at $\mathrm{RVb}$ minima and of the X-ray emission that may arise from accreted material.
\end{abstract}

Unified Astronomy Thesaurus concepts: Binary stars (154); Circumstellar matter (241); RV Tauri variable stars (1418); Spectral energy distribution (2129); Submillimeter astronomy (1647); X-ray astronomy (1810); Postasymptotic giant branch stars (2121)

Supporting material: data behind figure

\section{Introduction}

RV Tauri-type variable stars are $\mathrm{F}$ through $\mathrm{K}$ supergiants with luminosity classes between Ia and II. They extend the brightest part of the Type II Cepheid (i.e., RR Lyr, BL Her, W Vir, and RV Tau stars) instability strip on the H-R diagram, as well as the brightest part of their period-luminosity relation, with radial pulsation periods longer than 20 days (Soszyński et al. 2017). However, it has been shown that RV Tauri variables follow a steeper period-luminosity relation compared to their shorter-period Type II Cepheid counterparts (e.g., Bódi \& Kiss 2019).

The signature characteristic of RV Tauri variables are the alternating deep and shallow minima in their light curves: the time between a deep minimum and its successive shallower

\footnotetext{
${ }^{10}$ NASA MUREP Harriett G. Jenkins Pre-doctoral Fellow.

11 Fisk-Vanderbilt Master's-to-PhD Bridge Program.
}

Original content from this work may be used under the terms of the Creative Commons Attribution 4.0 licence. Any further distribution of this work must maintain attribution to the author(s) and the title of the work, journal citation and DOI. minimum is called the "fundamental" period; the time between two successive deep minima is the "formal" period. The alternation of deep and shallow minima is not strict in nature, as the light curves also show strong cycle-to-cycle variability, where the depths of the minima can vary randomly and may be attributed to low-dimensional chaotic behavior (e.g., Plachy et al. 2014, 2018, and references therein). In addition to pulsations, a photometric subgroup (RVb type) displays an additional, slower, periodic variation in mean brightness that ranges between 470 and 2800 days (Soszyński et al. 2017). There are $\sim 300$ identified RV Tauri variables in the Galaxy (Soszyński et al. 2020).

It has been established that most RV Tauri variables are a subclass of post-asymptotic giant branch (AGB) stars in binary systems surrounded by a dusty circumbinary disk (CBD), and there is argument for the companion to be an unevolved mainsequence star (van Winckel et al. 2009; Manick et al. 2019). The post-AGB primary is evolved from a low-to-intermediate-mass progenitor $\left(0.8-8 M_{\odot}\right)$, on their way to forming a planetary nebula, based on high luminosities $\left(\sim 10^{3}-10^{4} L_{\odot}\right)$, mass-loss history, and infrared excess (indicative of dust) in their spectral energy distribution (SED; Gehrz 1972; Evans 1985; Jura 1986; 
Alcock et al. 1998; van Winckel 2003). Groenewegen \& Jurkovic (2017) recently found mass estimates for RV Tauri variables in the Magellanic Clouds that showed either very high $\left(\gtrsim 1 M_{\odot}\right)$ or very low mass values $\left(\lesssim 0.5 M_{\odot}\right)$, which are in conflict with the standard single-star evolution of a post-AGB object, likely due to different evolutionary channels and perhaps revealing the effects of binarity. It may be that some of these post-AGB systems may evolve too slowly to ever become planetary nebulae (van Winckel 2017). Bódi \& Kiss (2019) showed similar mass estimate results to those of Groenewegen \& Jurkovic (2017), for a small sample of Galactic RV Tauri variables.

It was recently discovered that some of the less luminous RV Tauri variables are actually post-red giant branch (postRGB) stars, a class of objects that have similar spectroscopic stellar parameters to their post-AGB counterparts but appear at lower luminosities on the H-R diagram (Kamath et al. 2016; Manick et al. 2018). These objects are thought to have evolved off the RGB instead of the AGB as a result of binary interaction.

The CBDs around post-AGB (and post-RGB) binaries are described as second-generation "scaled-up" analogs to protoplanetary disks surrounding young stellar objects (YSOs; Hillen et al. 2017; van Winckel 2018). The near-IR excess observed in the SEDs of these bright post-AGB binaries, especially the RV Tauri variables, comes from hot dust close to the central source, where a disk-like nature of the near-IR emission has been confirmed by interferometric observations (Deroo et al. 2006). The mid- to far-IR excess then comes from cooler dust in the disk (de Ruyter et al. 2006; Hillen et al. 2015).

The CBDs are found to be relatively compact with sufficient angular momentum, in combination with the binary system's gravity, to settle and form the rotating disk from ejected material, especially from the huge mass loss after the AGB phase (up to $10^{-4} M_{\odot} \mathrm{yr}^{-1}$ ) of the evolved primary (Bujarrabal et al. 2013). Keplerian rotation of the circumbinary material around these stars has been resolved by narrow $\mathrm{CO}$ line profiles, as well as the presence of large grains with a high degree of crystallinity, indicating longevity and stability, that form these disks (Gielen et al. 2011) with diameters ranging between $\sim 100$ and 2000 au (Bujarrabal et al. 2005, 2013, 2018).

The slower photometric phenomenon from the $\mathrm{RVb}$ subset of RV Tauri systems (and in other post-AGB binaries) has been attributed to an extrinsic variable extinction. An inclined CBD can shadow the primary at certain phases of its orbit (e.g., Manick et al. 2017), blocking the light, and causing extinction and scattering along our line of sight. Vega et al. (2017) used ultraprecise flux measurements from the Kepler telescope and found that the decrease in pulsation amplitude of DF Cyg (the only RV Tauri system in Kepler's original field of view) perfectly tracked the decrease in flux from its $\mathrm{RVb}$ oscillation, showing that the long-term minima are due to the disk obscuring the pulsating primary. Kiss \& Bódi (2017) further extended this study to all known RVbs in the Galaxy (by also adopting flux units instead of the inverse logarithmic magnitude system) to confirm that all RVbs displayed the correlation found in DF Cyg. Furthermore, by using interferometric observations, Kluska et al. (2019) have found that the $\mathrm{RVb}$ sources in their sample have high inclinations, implying a very high disk scale height and allowing the disk-shadowing interpretation to be correct.
The presence of disks has also been linked to the phenomenon known as depletion, which is found in postAGB binaries. Depletion is a systematic underabundance of refractory elements, in the photospheres of post-AGB stars, that correlates with the condensation temperature of an element (Giridhar et al. 2000). In order to get a depleted photosphere, the stellar radiation pressure on circumstellar material separates dust grains (containing refractory elements) from the volatilerich gas that gets reaccreted onto the stellar surface. Since dust grains experience a much larger radiation pressure, they do not get accreted. Depletion is thought to be caused by accretion of metal-poor gas from the CBD (Waters et al. 1992). Even though the presence of a disk seems to be needed, it is not a sufficient condition for depletion to occur since not all postAGB binaries with disks are depleted (Gezer et al. 2015).

Though it is clear that binarity plays a very important role in the dynamics and evolution of post-AGB systems, the details of binary interaction processes are still not well understood (van Winckel 2018) and have been an important topic of investigation. New advances on the discovery of high-velocity outflows in post-AGB binaries have brought to light unique orbital phase-dependent variations in the $\mathrm{H} \alpha$ profiles and have proven to be rather common in post-AGB binaries (Gorlova et al. 2012, 2014, 2015; Bollen et al. 2017, 2019, 2020). Studies associate this phenomenon with binary interaction and show that these high-velocity outflows (i.e., a bipolar jet) are launched by an accretion disk around the companion (i.e., circumcompanion accretion disk) that produces a $\mathrm{P}$ Cygni profile from the $\mathrm{H} \alpha$ emission line, as the jet from the companion transits the bright post-AGB primary. Based on accretion models for two well-sampled post-AGB binaries, Bollen et al. (2020) concluded that the CBD is likely feeding a circumcompanion accretion disk. This agrees with the observed depletion patterns of refractory elements in post-AGB binaries (Oomen et al. 2020). Manick et al. (2019) have already shown similar $\mathrm{H} \alpha$ variations that point to the presence of jets for two RV Tauri systems: RV Tau and DF Cyg.

In the context of the recent developments in the field of postAGB binaries, we report new observations that may provide insight into how binarity plays a role in these systems. In this paper we present the most comprehensive, multiwavelength analysis to date of a classical $\mathrm{RVb}$ variable: $\mathrm{U}$ Monocerotis (U Mon). The analysis includes an X-ray detection-never before reported for any RV Tauri system-and an unprecedented photometric time series spanning 130 yr. Our results focus on consolidating several characteristics displayed in previous measurements, such as the very long time baseline photometry. We introduce recent space- and ground-based data collected for U Mon from the XMM-Newton X-ray satellite and the Submillimeter Array (SMA), respectively.

We give an introduction to the U Mon system in Section 2 and describe each data set in Section 3. We present our results in Section 4, including evidence for an even longer-term secular variation in the U Mon light curve that may recur every $\sim 60 \mathrm{yr}$ and that may represent a persistent feature in the U Mon CBD; a refined determination of the disk's inner hole, which appears to coincide with the size of the binary orbit; a new characterization of the binary companion star; and evidence for its possible interaction with the CBD's inner edge. In Section 5 we discuss the implications of our findings and present $\mathrm{U}$ Mon as a template for the discovery of X-rays in the RV Tauri 
Table 1

Observed and Derived Physical Properties for the U Mon System Used in Our Analysis

\begin{tabular}{lcc}
\hline \hline Properties & Value & Reference \\
\hline Spectral type & K0Ibpv & 1 \\
{$[\mathrm{Fe} / \mathrm{H}]$} & -0.8 & 2 \\
{$[\mathrm{C} / \mathrm{O}]$} & 0.8 & 2 \\
$T_{\text {eff }}$ & $5000 \mathrm{~K}$ & 2 \\
Distance & $1111_{-102}^{+137} \mathrm{pc}$ & $3^{\mathrm{a}}$ \\
Radius & $100_{-13.2}^{+18.9} R_{\odot}$ & 3 \\
Luminosity & $5480_{-882}^{+1764} L_{\odot}$ & 3 \\
Mass & $2.07_{-0.9}^{+1.4} M_{\odot}$ & $3^{\mathrm{b}}$ \\
Pulsation formal period & 91.48 days & 4 \\
RVb long-term period & 2451 days & 4 \\
\hline
\end{tabular}

\section{Binary}

Inclination

Orbital period

$e$

$T_{0}$

$\omega$

$K_{1}$

Mass function

Mass $_{\text {Companion }}$

Semimajor axis

\section{Disk}

Diameter

Dust mass

Inner-disk edge radius

References. (1) He et al. 2014; (2) Giridhar et al. 2000; (3) Bódi \& Kiss 2019; (4) Kiss \& Bódi 2017; (5) Oomen et al. 2018; (6) this study.

a Based on Gaia measurements. However, see note in text (Section 2.2) regarding the Gaia parallax accuracy and uncertainty.

b Average of the values from the Groenewegen \& Jurkovic (2017) periodluminosity-mass relationships adopted in Bódi \& Kiss (2019).

subclass of post-AGB binaries. Finally, we summarize our conclusions in Section 6.

\section{The U Monocerotis System}

In this section we provide an observational overview of the U Mon system and review the physical properties that have been determined from previous studies. Table 1 summarizes the observed and derived physical properties that we use in our analysis.

\subsection{U Mon as an RVTauri Variable of RVb Type}

U Mon, a yellow supergiant variable (KOIbpv; He et al. 2014), is located at a distance of $1111_{-102}^{+137}$ pc (Bódi \& Kiss 2019). It is the second-brightest RV Tauri variable (after $\mathrm{R}$ Sct) with a magnitude range of 5.45-7.67 in $V$ (Watson et al. 2006), mean color index $B-V=1.05$ mag at $\mathrm{RVb}$ brightness maximum, $1.11 \mathrm{mag}$ at RVb minimum (Pollard et al. 1996), and a metallicity of $[\mathrm{Fe} / \mathrm{H}]=-0.8$ (Giridhar et al. 2000). Its formal pulsation period (deep + shallow minima cycle) is 91.48 days, and its fundamental period is 45.74 days. U Mon also exhibits a long-term periodic modulation in mean brightness ( $\mathrm{RVb}$ phenomenon) with a period of $\sim 2451$ days (Kiss \& Bódi 2017), where the large-amplitude difference in mean brightness is $\sim 3$ mag.

\subsection{U Mon as a Binary Star System}

With BVRI photometry and high-resolution spectra, Pollard $\&$ Cottrell (1995) concluded that U Mon is an eccentric binary ( $e=0.43$ ) exhibiting a radial velocity amplitude of $30 \mathrm{~km} \mathrm{~s}^{-1}$ (full amplitude) and an orbital period of $\sim 2597$ days, which is similar to its photometric $\mathrm{RVb}$ period, as found in other $\mathrm{RVb}$ systems (Manick et al. 2017).

Oomen et al. (2018) recently updated the orbital properties of the UMon binary system using data from the HERMES spectrograph on the $1.2 \mathrm{~m}$ Mercator telescope. They found an orbital period of $2549 \pm 143$ days. By assuming a typical postAGB mass for the primary of $0.6 M_{\odot}$ and an inclination of $75^{\circ}$, they estimated a projected semimajor axis of $3.38 \pm 0.31 \mathrm{au}$, a mass function of $0.79 \pm 0.18 M_{\odot}$, and hence a minimum mass of $1.64 M_{\odot}$ for the companion. However, most recently Bódi \& Kiss (2019) used Gaia (DR2; Gaia Collaboration et al. 2018) data and two different period-luminosity-mass-temperaturemetallicity relations derived by Groenewegen \& Jurkovic (2017; based on hydrodynamical atmosphere modeling of fundamental-mode pulsators from Bono et al. 2000; Marconi et al. 2015), to empirically infer the mass of the post-AGB star in $\mathrm{U}$ Mon to be between 2.00 and $2.13 M_{\odot}$. For our analysis we adopt the mean of these last two values (Section 4.2).

We note that the Gaia distance is likely biased by the orbital movement of the binary. The renormalized unit weight error (RUWE) for U Mon (RUWE = 2.4) indicates a poor single-star solution. High RUWE can be caused by a variety of factors, including resolved components in U Mon (see Belokurov et al. 2020). The astrometric excess noise $\left(\epsilon_{\mathrm{Gaia}}\right)$ for U Mon in the DR2 catalog is 0.40 mas. This parameter is the excess uncertainty that must be added in quadrature to the formal uncertainties to obtain a statistically acceptable astrometric solution in the DR2 pipeline (Lindegren et al. 2012). Gandhi et al. (2020) considered using $\epsilon_{\text {Gaia }}$ as a proxy for the expected astrometric orbital wobble $(\omega)$ to help identify potential X-ray binaries. Given that the Gaia DR2 observations $^{12}$ occurred over only $\sim 25 \%$ of the orbit of the U Mon system, the maximum expected orbital wobble for our orbit solution for U Mon at a distance of $1.1 \mathrm{kpc}$ is $\sim 0.3$ mas, which is comparable to the reported $\epsilon_{\text {Gaia }}$ value, suggesting that the excess uncertainty could be mostly due to orbital motion. Indeed, the newly updated Gaia Early Data Release 3 parallax ${ }^{13}$ of $1.28 \pm 0.12$ mas differs from the Gaia DR2 parallax by 0.36 mas, again very similar to $\epsilon_{\mathrm{Gaia}}$. Therefore, if we adopt $\epsilon_{\mathrm{Gaia}}$ as the uncertainty in the parallax measurement, then the distance to U Mon ranges between $\sim 770 \mathrm{pc}$ and $\sim 2 \mathrm{kpc}$. However, using the same period-luminosity-mass relations by Groenewegen \& Jurkovic (2017) as were used originally by Bódi \& Kiss (2019), we find that the stellar and orbital quantities in Table 1 do not change significantly relative to the already-quoted uncertainties. Therefore, we adopt the nominal distance of $1.1 \mathrm{kpc}$ as in Bódi \& Kiss (2019).

\subsection{The UMon Circumbinary Disk}

As with many other RV Tauri variables in the literature, the U Mon binary star system is surrounded by a CBD. Bujarrabal et al. (2013) estimated the size of the UMon CBD to be $\sim 300$ au using observations at the IRAM $30 \mathrm{~m}$ telescope in 2012-2013. Though they did not detect the Keplerian rotation

\footnotetext{
${ }^{12}$ Section 1.3.1 Time coverage: https://gea.esac.esa.int/archive/documentation/ GDR2/.

${ }^{13}$ Gaia EDR3 was released on 2020 December 01.
} 


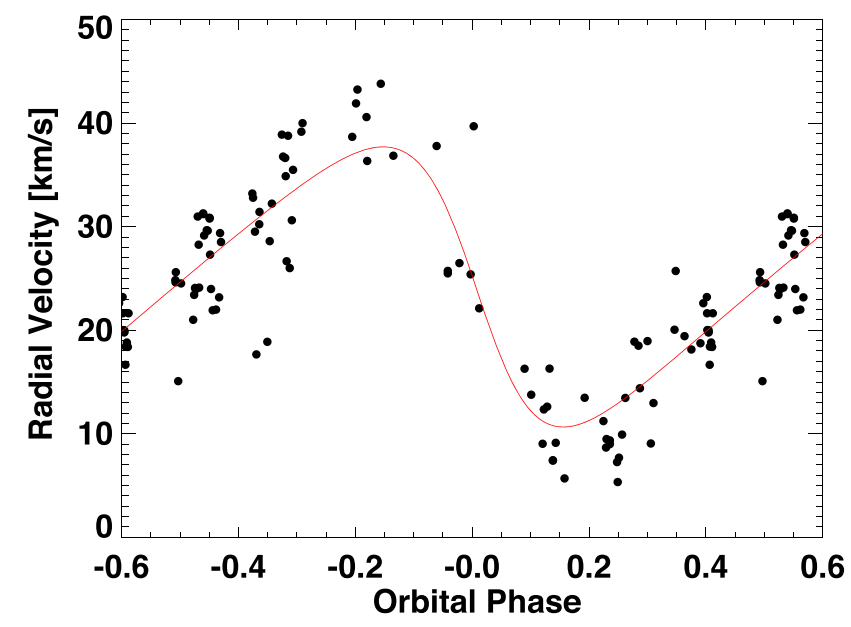

Figure 1. Single-lined orbit solution for U Mon as fit to the radial velocity observations of Oomen et al. (2018).

of the CBD, they derived an upper limit for the molecular gas mass $\left(<9 \times 10^{-4} M_{\odot}\right)$ from their ${ }^{13} \mathrm{CO} J=1-0$ data.

In addition, Kluska et al. (2019) were able to reproduce their recent Very Long Baseline Interferometer (VLBI)/PIONIER near-IR $(H$-band) observations for U Mon by using their most complex model that includes a binary and inner ring. They suggested an an inner-disk diameter of $\sim 5.9 \mathrm{au}$.

\subsection{Magnetic Activity in UMon}

Sabin et al. (2015) were the first to find magnetic fields at the surface of U Mon. The Stokes $Q$ and $U$ profiles were observed at $\mathrm{RVb}$ phase $\sim 0.63$ and the Stokes $V$ profile at phase $\sim 0.84$ in Figure 6. They analyzed high-resolution spectropolarimetric ESPaDOnS (CFHT) data and found a clear Zeeman signature in the Stokes $V$ profile. They measured a longitudinal magnetic field (i.e., in the line of sight) of $10.2 \pm 1.7 \mathrm{G}$ in the photosphere of UMon. Although the sample is poor, this is to date the strongest surface field directly detected for a postAGB star. The Stokes $Q$ and $U$ profiles indicated the presence of shocks, and the authors suggested the possible amplification of the magnetic field due to the atmosphere dynamics.

\section{Data \\ 3.1. Radial Velocity Observations}

We adopted radial velocity observations of U Mon, corrected for the effects of surface pulsations, reported by Oomen et al. (2008, available on VizieR). Most photometric RVb periods of RV Tauri variables are similar to the orbital periods of their binaries (Manick et al. 2017). To calculate a new orbital solution for the binary in U Mon, we fixed the orbital period at 2451 days (U Mon's photometric RVb period; Bódi \& Kiss 2019), and we refit the data with a single-lined spectroscopic, Keplerian binary orbit model using PHOEBE (Prša et al. 2016). The resulting fit is shown in Figure 1, and the fit parameters are summarized in Table 1 . We discuss our findings in Section 4.2.

\subsection{Light-curve Observations}

We use two sources of long-term photometric monitoring of $\mathrm{U}$ Mon in order to explore secular changes in the U Mon light curve on timescales of decades or longer. The full light-curve data set is represented in Figure 2.

\subsubsection{AAVSO}

The American Association of Variable Star Observers (AAVSO) is a global network of amateur and professional astronomers dedicated to monitoring variable stars. The earliest observation of UMon in the AAVSO archive was made by Ernest E. Markwick on 1888 December 25 (JD 2,410,997.0), and after a few observations there followed a 49 yr gap between 1896 and 1945. More regular monitoring began in the mid-1940s and continues to the present day. We downloaded all (including "discrepant"-flagged) $\mathrm{Mag}_{V}$ data from the AAVSO database up to 2020 May 26. We chose to keep discrepant data since we are not focused here on the pulsation variability. We also wanted to make sure we had the data between 1888 and 1896 (which are all marked discrepant) because they capture a long-term RVb cycle; these data are nonetheless useful for our purposes and fill a gap in time that is otherwise unavailable. We only excluded $36 \mathrm{Mag}_{V}$ data points tagged as upper limits.

\subsection{2. $\mathrm{DASCH}$}

The Digital Access to a Sky Century at Harvard (DASCH) survey is an ongoing effort to digitize about 0.5 million photographic plates covering the northern and southern sky from 1880 to 1985 (Grindlay et al. 2009).

Data for U Mon were released in the DASCH Data Release 6 (DR6). We downloaded the light curve of UMon from the DASCH Light Curve Access pipeline website ${ }^{14}$ using the default search radius of $5^{\prime \prime}$. Since the majority of the Harvard plates are close to Johnson $B$, we chose the data from the APASS $B$ photometric calibration catalog, which yields the most accurate photometry (Tang et al. 2013).

The DASCH (APASS $B$-band catalog) light curve of U Mon (ID: T540046991) contained 3824 magnitude data points from approximately 3436 plates at the time of download. The light curve has a mean magnitude of 8.77 in $B$ and a baseline going back to 1888 January 25 (JD 2,410,661.7) with occasional gaps, the largest one being the "Menzel" gap in the 1950s and 1960s, when the plate-making operation was halted by the Harvard Observatory director at the time owing to financial concerns. We excluded DASCH data points that had estimated errors of the locally corrected magnitude measurement (magcal_local_rms) values greater than $0.6 \mathrm{mag}$ (which included magcal_local_rms values set to 99.0; these are tagged magnitudes from images dimmer than the limiting value of the image). We also excluded magnitude-dependent corrected magnitude (magcal_magdep) values brighter than 2.0 and dimmer than 11.0 (which were well away from the mean magnitude of the overall light curve).

\subsubsection{Combining AAVSO and DASCH Light-curve Data}

To analyze the AAVSO and DASCH data sets together, we converted the light curves to flux units using arbitrary zeropoints (ZPs). For AAVSO we adopted ZP $=25$ (Kiss \& Bódi 2017). Because the DASCH effective bandpass is different from the AAVSO $\mathrm{Mag}_{V}$ bandpass, we experimented with ZP values for DASCH to empirically determine a scaling

\footnotetext{
${ }^{14}$ http://dasch.rc.fas.harvard.edu/lightcurve.php
} 


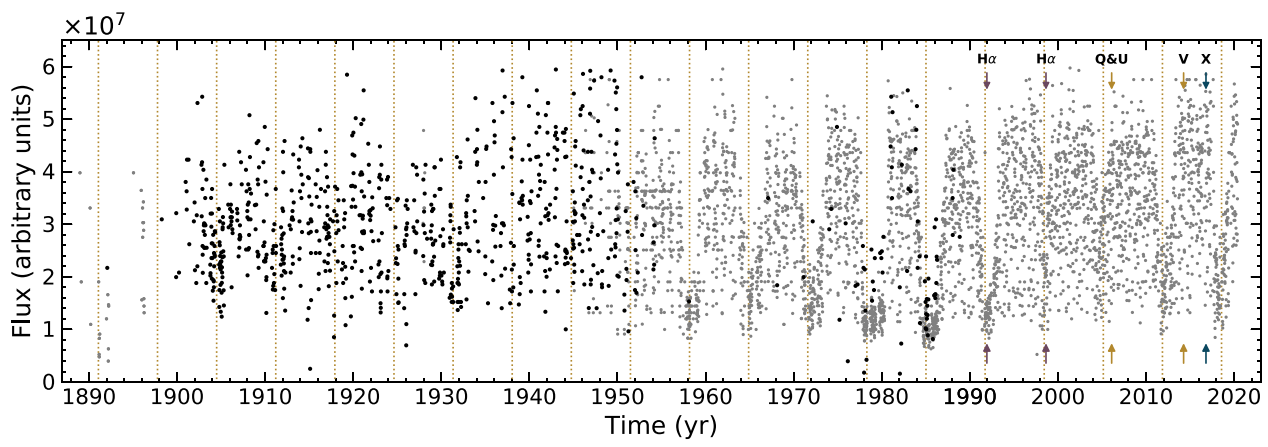

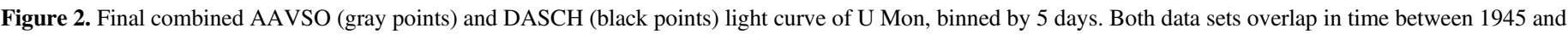

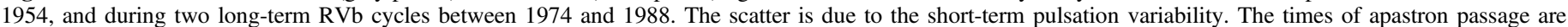

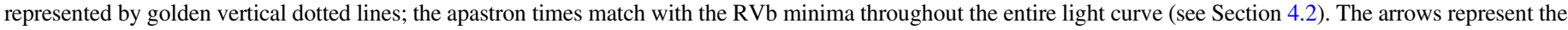

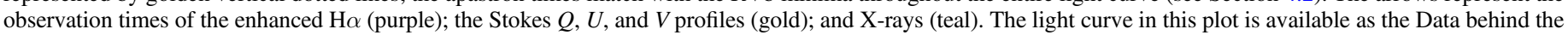
Figure (DbF).

(The data used to create this figure are available.)

factor and ensure a proper match of the AAVSO and DASCH data during two RVb cycle amplitudes (between 1974 October and 1988 August), where the AAVSO and DASCH data sets overlap to the greatest degree in time. We adopted $\mathrm{ZP}=27.5$ for DASCH as the best fit but found that we also had to scale the DASCH fluxes by a factor of 1.21 and then subtract $1.1 \times 10^{7}$ flux units to match the two overlapping RVb cycles (see Figure 3). After we converted to arbitrary flux units, we made another cut to exclude data above $\sim 6 \times 10^{7}$ flux units and $\leqslant 0$.

The final combined AAVSO+DASCH light curve is shown in Figure 2. It is an impressive data set, spanning the period from $\sim 1890$ to $\sim 2020$. The light curve is binned by 5 days to average out errors of individual observations and to reduce phase smearing due to the binning (Kiss \& Bódi 2017). What seems to be leftover scatter is actually due to pulsations of different amplitudes, as well as large gaps in the data (see, e.g., Figure 3). Several features are present in the secular changes of the light curve on timescales of decades, and we discuss this in Section 4.1.

\subsection{SMA Observations}

The SMA is an interferometer, composed of eight $6 \mathrm{~m}$ dishes, that observed U Mon on three occasions. On 2018 February 1, we observed the source in the subcompact array configuration with projected baselines of 7-49 $\mathrm{m}$ and covered two frequency ranges at 223.6-231.6 GHz and 239.6-247.5 GHz. On 2018 October 2, observations were made at higher frequencies, from 328.8 to $360.8 \mathrm{GHz}$, and at longer baselines of 6-70 m. On 2019 March 15, we finally observed UMon in the very extended SMA configuration (VEX) with baselines from 32 to $514 \mathrm{~m}$. The covered frequency ranges were $209.1-212.8 \mathrm{GHz}, 214.9-216.7$ GHz, 225.1-226.9 GHz, 229.2-238.8 GHz, 332.8-340.8 GHz, and $348.8-356.8 \mathrm{GHz}$. The spectral coverage in the VEX observations is smaller than on the earlier dates owing to a nonfunctional quadrant of the SMA correlator SWARM (Primiani et al. 2016) at that time. All eight SMA antennas were used for our first two observing runs except for observations in VEX at low frequencies for which only six antennas had properly functioning receivers.

Because the target was observed mainly as a filler project, ${ }^{15}$ the final $u v$ coverage is suboptimal, in particular, very

\footnotetext{
15 http://sma1.sma.hawaii.edu/call_filler.html
}

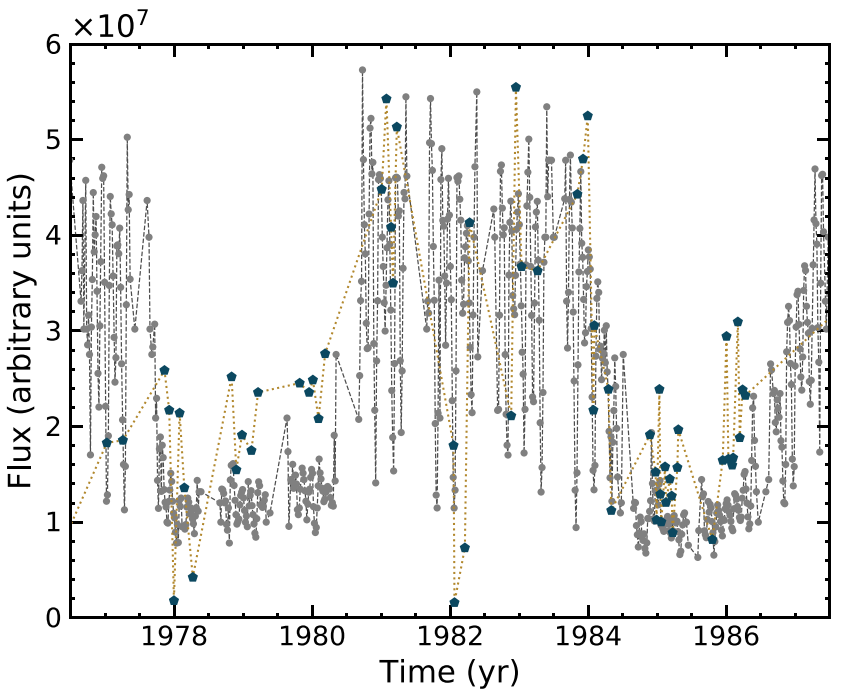

Figure 3. Two long-term RVb minima where the DASCH (teal pentagons) and AAVSO data (gray points) overlap the most in time. The gray dashed line on the AAVSO points (yellow dotted line for DASCH), though affected by the 5-day binning of the data, mainly highlights the pulsation variation of $\mathrm{U}$ Mon. Pulsation amplitudes during the RVb maxima may extend to as low as the mean magnitude at RVb minima, while the pulsations during RVb minima are always smaller in amplitude, showing the effect of disk obscuration of the pulsating post-AGB.

inhomogeneous at higher frequencies. The complex antenna gains were calibrated in all observing runs by observations of quasars J0730-116 and J0725-009. The bandpass was calibrated using long integrations of quasars 3C 279 and 3C 84. An absolute flux scale was established by observations of Uranus (on February 1), Neptune (on October 2), and Callisto (in 2019). The calibration was performed in MIR software using standard procedures. ${ }^{16}$ Further data processing, including imaging, was performed in CASA (McMullin et al. 2007).

Calibrated visibilities measured in the different array configurations were combined within the two atmospheric windows near $345 \mathrm{GHz}(\sim 0.8 \mathrm{~mm})$ and $230 \mathrm{GHz}(1.3 \mathrm{~mm})$ and their weights rescaled to the actual noise levels. This resulted in continuum sensitivities of $\sigma_{345}=4.0 \mathrm{mJy} \mathrm{beam}^{-1}$ and $\sigma_{230}=0.89 \mathrm{mJy}$ beam $^{-1}$ at beam sizes of $4 . \prime 0 \times 2$ !" 4 and 0 ! $9 \times 0$ ". 6 , respectively. These beam sizes correspond to

\footnotetext{
16 http://www.cfa.harvard.edu/ cqi/mircook.html
} 

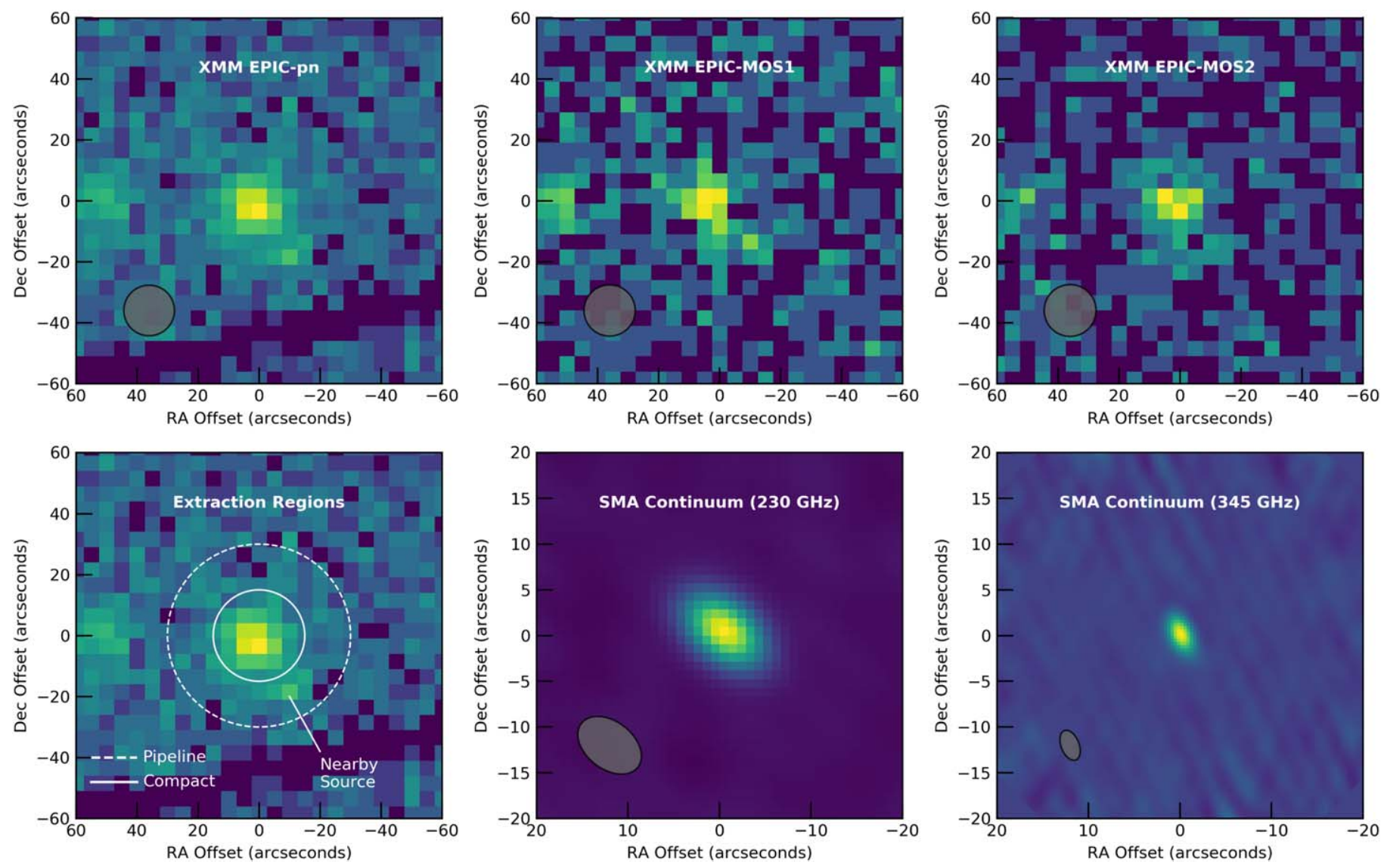

Figure 4. Top panels: XMM EPIC-pn, EPIC-MOS1, and EPIC-MOS2 images with a clear detection of X-rays for U Mon. Bottom left panel: EPIC-pn image annotated with the pipeline extraction vs. our compact extraction region excluding the nearby source near U Mon. Bottom middle and right panels: U Mon's SMA continuum images at $230 \mathrm{GHz}(1.3 \mathrm{~mm})$ and $345 \mathrm{GHz}(870 \mu \mathrm{m})$, respectively. The respective beams are included in the lower left corners in dark gray.

Table 2

New Flux Measurements for U Mon

\begin{tabular}{lccr}
\hline \hline Wavelength $(\mu \mathrm{m})$ & Flux $(\mathrm{mJy})$ & Date & System \\
\hline 0.212 & $23.27 \pm 0.038$ & 2016 Oct & XMM-OM:UVM2 \\
0.231 & $16.30 \pm 0.030$ & 2016 Oct & XMM-OM:UVW2 \\
869.4 & $173.3 \pm 6.5$ & 2018 Feb, Oct; 2019 Mar ${ }^{\mathrm{a}}$ & SMA \\
1313 & $70.2 \pm 8.5$ & 2018 Feb, Oct; 2019 Mar ${ }^{\mathrm{a}}$ & SMA \\
\hline
\end{tabular}

Note.

${ }^{\mathrm{a}}$ Combined observations.

natural weighting of visibilities. In both bands the continuum source was readily detected at flux levels listed in Table 2; see also Figure 4. No spectral lines were detected with an rms noise level of $39.3 \mathrm{mJy} \mathrm{beam}^{-1}$ near the frequency of the CO $J=2-1$ line and at a $3.9 \mathrm{~km} \mathrm{~s}^{-1}$ spectral binning, which is consistent with the nondetection reported by Bujarrabal et al. (2013). We discuss U Mon's submillimeter emission in Section 4.5.

\subsection{XMM-Newton Observations}

U Mon was observed by the X-ray Multiple Mirror (XMMNewton) observatory on 2016 October 23 for $58.3 \mathrm{ks}$ (RVb phase $\sim 0.23$ in Figure 6). The XMM-Newton observations include 15 imaging exposures: three X-ray images on the EPIC-pn $(55.3 \mathrm{ks})$, EPIC-MOS1 (57.0 ks), and EPIC-MOS2 (56.9 ks) detectors, and 12 exposures (each $\sim 2.2-4.4 \mathrm{ks}$ ) with the Optical Monitor (OM) using the UVW1, UVM2, and UVW2 filters. Representative images are shown in Figure 4. U Mon is detected in all imaging exposures. Additionally, there are two grating dispersed exposures in X-ray emission on the RGS1/2 (57.2 ks), but the dispersed spectrum is not detected. The large source region used to generate the pipeline products leads to confusion and blending with a nearby source (see bottom left panel in Figure 4). We re-extracted the X-ray spectra and light curves using a smaller source region with a radius of $15^{\prime \prime}$ and a source-free background region near the source. The extracted spectral products were corrected for the reduced extraction region by accounting for the encircled energy fraction in the response files.

\subsection{Spectral Energy Distribution Data}

To construct U Mon's SED, we downloaded available archival photometric data from VizieR. We did not take into account the phase of observations (i.e., either at maximum/minimum 


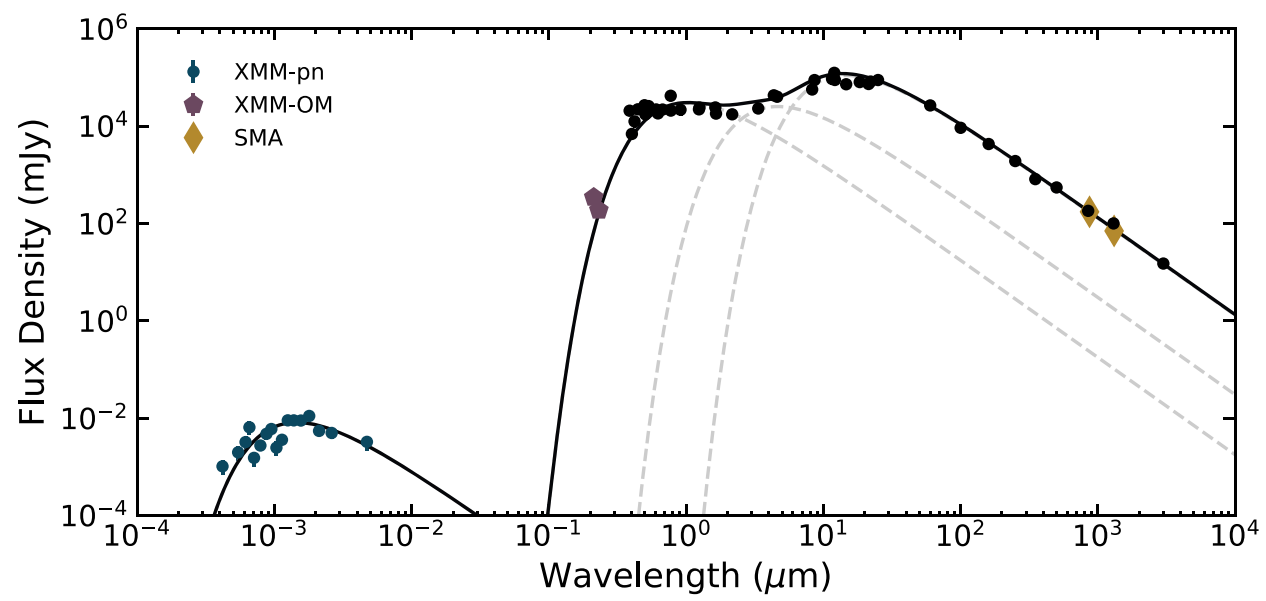

Figure 5. SED for U Mon. Representative archival data (black points) were collected from VizieR and the Herschel archives, as well as the $850 \mu \mathrm{m}$ measurement from de Ruyter et al. (2005) and the 1.3 and $3 \mathrm{~mm}$ measurements from Sahai et al. (2011). We present the new XMM-pn spectrum in teal points, the XMM-OM data in pentagons, and the SMA data in diamonds. The scatter in the archival data is probably due to intrinsic (pulsation or RVb) variability of U Mon at the time of observation. Note: error bars are smaller than the symbols.

pulsation or long-term RVb brightness). We included HerschelPACS/SPIRE measurements (downloaded from the ESA Herschel Science Archive ${ }^{17}$; PI: C. Gielen), the $850 \mu \mathrm{m}$ flux value reported by de Ruyter et al. (2005) taken with the Submillimetre Common-User Bolometer Array (SCUBA) at the James Clerk Maxwell Telescope (JCMT), and finally millimeter fluxes from Sahai et al. (2011) taken at the Combined Array for Research in Millimeter-wave Astronomy (CARMA).

The new observations we contribute in this paper are submillimeter and UV/X-ray from the SMA and XMM-Newton, respectively. Our SMA $870 \mu \mathrm{m}$ and $1.3 \mathrm{~mm}$ values are similar to those reported by de Ruyter et al. (2005) and Sahai et al. (2011), respectively. The XMM-OM magnitudes for UMon were converted to flux density (Jy) using the ZPs from Mason et al. (2001). To represent the XMM EPIC-pn spectrum, we unfolded the instrumental response from the spectral data assuming the best-fit model determined using the X-ray Spectral Fitting Package (XSPEC ${ }^{18}$; Arnaud 1996), dereddened the spectral data (see Section 4.3), and then converted from photon flux densities to Jy.

Figure 5 shows the SED for U Mon, and Table 2 lists our new flux measurements. In Section 4.3 we discuss the blackbody models we used on the SED.

\section{Results}

\subsection{Secular Variations of the U Mon Light Curve over the Past Century}

The long-term RVb cycles of the U Mon light curve are more salient in the AAVSO data, and we can estimate the duration of the maxima more easily than the minima. Overall, the RVb phenomenon in UMon changes significantly from cycle to cycle. The RVb maxima range between $\sim 3.3$ and $4.8 \mathrm{yr}$, whereas the $\mathrm{RVb}$ minima differ even more, from several months to the longest minimum lasting $\sim 2.5 \mathrm{yr}$ (e.g., between 1977.8 and 1980.5; see Figure 3).

In Figure 6 we explore longer-term secular changes in the light curve, enabled by unprecedented coverage spanning $\sim 130 \mathrm{yr}$. We

\footnotetext{
17 http://archives.esac.esa.int/hsa/whsa/

18 http://heasarc.gsfc.nasa.gov/xanadu/xspec/
}

binned both data sets by U Mon's fundamental pulsation period (45.74 days) to focus on the RVb behavior. We plot the DASCH data in black, the AAVSO data in gray, and the DASCH data that distinctly overlap the two RVb cycles during 1975-1988 as teal squares. We also define golden squares that are AAVSO data sporadically overlapping with DASCH data before 1951, and AAVSO data corresponding to what might be times of partially obscured RVb minima ( 1997-2008). The top panel is the binned light curve in time, the bottom panel is the binned light curve phased by the RVb period of 2451 days, and the flux is normalized to be unity at the base level. We fit parabolas to the data at phases of $0.5 \pm 0.3$, to get a cleaner visual sense of how the $\mathrm{RVb}$ minima compare for the various subsets.

We find that the AAVSO data (gray points) and the DASCH overlap data (teal squares) have nearly identical RVb minima. Similarly, we see that the older DASCH data (black points) and the golden-square AAVSO data have nearly identical RVb minima, and the latter minima are about a factor of 2 less deep. In other words, it appears from the historical record that the $\mathrm{RVb}$ variations-which have come to be associated with a highly inclined disk shadowing the primary at certain orbital phases - have largely disappeared at least twice in the past, with a timescale of $\sim 75 \mathrm{yr}$, from the middle of the black points to the latest golden squares in Figure 6.

Another way to examine this is shown in Figure 7, in which the disappearance of the $\mathrm{RVb}$ variation, due to the large scatter in the data points, is more obvious for two $\mathrm{RVb}$ minima in 1938 and 1944 and then again in 1998 and 2005, with a timescale between them of $\sim 60.4 \mathrm{yr}$, which we note is nine times the $\mathrm{RVb}$ long period. To be clear, the $\mathrm{RVb}$ minima are short during these cycles ( $\sim$ several months, as opposed to years), and they "disappear" relative to the large scatter/ pulsation variations from the (particularly longer) maximum state at either side of $\mathrm{RVb}$ minima. We further discuss the interpretation of this phenomenon in Section 5.1.

\subsection{Orbital Properties of the UMon Binary Star System}

From the orbit fit in Section 3.1, we obtained orbital parameters (listed in Table 1) that are within the errors of the values consistent with Oomen et al. (2018), including a new periastron time of $T_{0}=2,452,203 \pm 17$ days. Adding half 

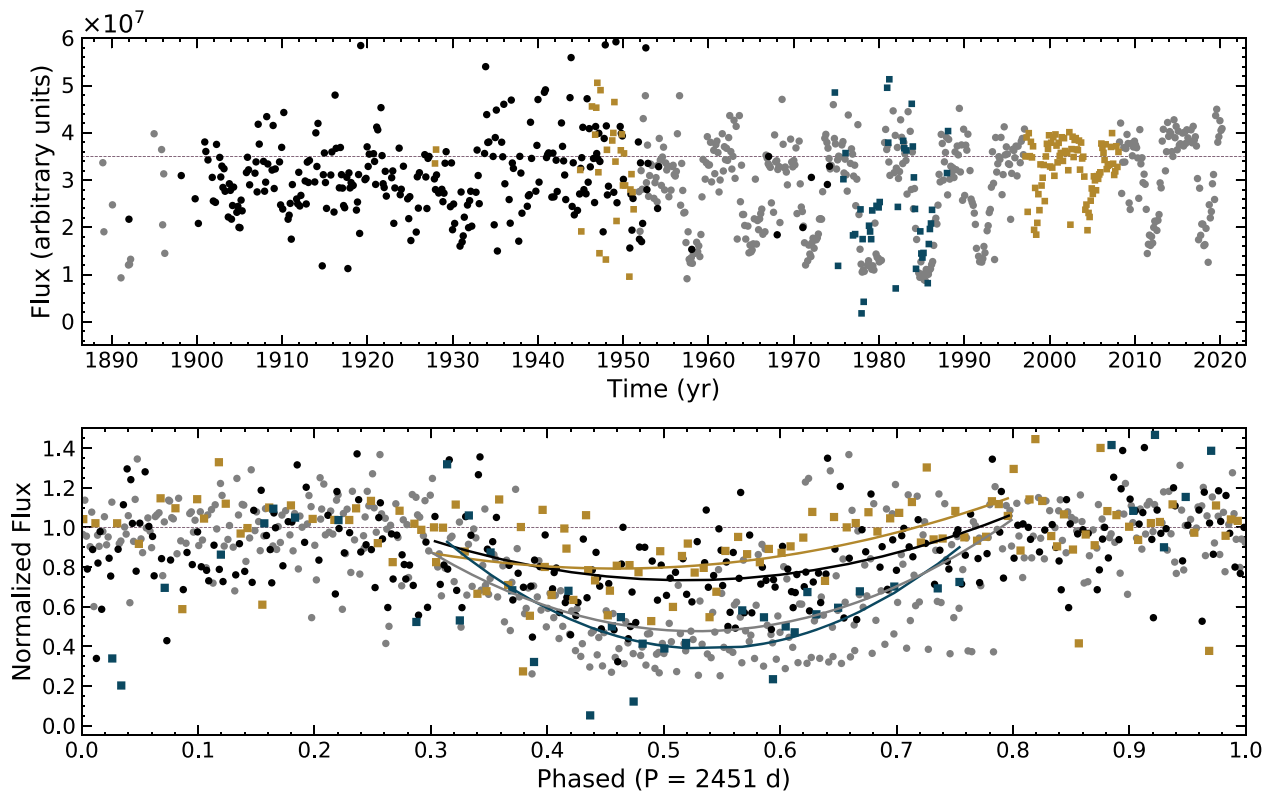

Figure 6. Analysis of secular variations in the U Mon light-curve data. Top: light curve binned by the fundamental period of 45.74 days. The black points are DASCH data, the gray points are AAVSO data, the DASCH data overlapping two distinct RVb cycles are shown as teal squares, and the golden squares define AAVSO data sporadically overlapping DASCH data before 1951 and data corresponding to what might be times of partially obscured minima ( 1997-2008). The dashed line is the mean flux at $\mathrm{RVb}$ maximum $\left(3.5 \times 10^{7}\right.$ flux units). Bottom: light curve phase-folded on the 2451-day RVb period of the system with flux normalized at unity at the base level (dashed line is the same as the top panel). See Section 4.1 for a discussion of the curves.

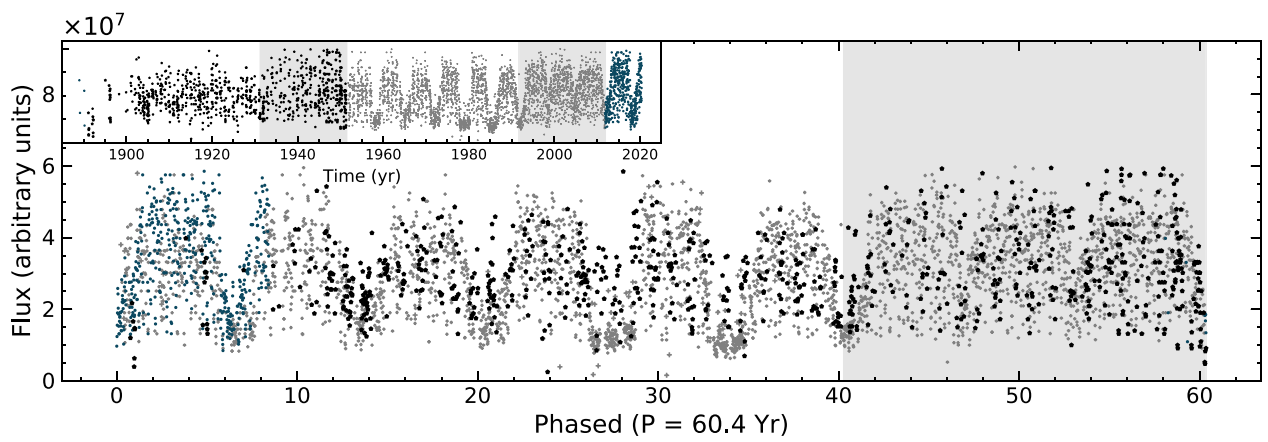

Figure 7. Light curve of U Mon phased at $60.4 \mathrm{yr}$ (nine RVb cycles). The inset shows the full light curve divided in color distinguishing the two 60.4 yr cycles in the data (black points represent the first $60.4 \mathrm{yr}$ cycle; gray points, second cycle). The start of a third cycle is shown as teal points. The RVb cycles highlighted in light gray include long ( $\lesssim 4.5 \mathrm{yr}$ ) RVb maxima and short $\mathrm{RVb}$ minima (phase $\sim 47 \mathrm{yr}$ and $\sim 53 \mathrm{yr}$ ) that have durations of only several months based on the AAVSO data.

(1225 days) of the fixed orbital period to the periastron time gives an apastron at $\mathrm{JD}=2,453,428$ days. Multiples of the orbital period with the apastron time give all apastron events (denoted as golden vertical dashed lines in Figure 2) that roughly align with the RVb minima throughout U Mon's entire $\mathrm{DASCH}+\mathrm{AAVSO}$ light curve.

Mass estimates for U Mon range from a fiducial post-AGB mass average of $0.6 M_{\odot}$ (Gezer et al. 2015; Manick et al. 2017; Weidemann 1990) to most recent values of 2.00 and $2.13 M_{\odot}$ reported by Bódi \& Kiss (2019).

To estimate a value for the companion's mass $\left(M_{2}\right)$, we used the average value from Bódi \& Kiss (2019) for the mass of the post-AGB $\left(M_{1}=2.07 M_{\odot}\right)$ in Equation (1) (the binary mass function for an eccentric orbit) and solved for $M_{2}$ :

$$
f(m)=\frac{P K_{1}^{3}}{2 \pi G}\left(1-e^{2}\right)^{\frac{3}{2}}=\frac{M_{2}^{3}}{\left(M_{1}+M_{2}\right)^{2}} \sin ^{3} i .
$$

Using the recalculated radial velocity curve, and keeping the orbital inclination maximum limit of $75^{\circ}$ (Oomen et al. 2018), we find the mass for the companion to be $2.2_{-0.75}^{+1.0} M_{\odot}$; this mass range corresponds to an F-type or A-type star on the main sequence. Then, by using Kepler's third law, we derived a value of $5.78_{-1.4}^{+2.7}$ au for the semimajor axis. These parameters are reported in Table 1.

\subsection{Spectral Energy Distribution}

We dereddened the flux data between 0.2 and $3.3 \mu \mathrm{m}$ using functions contained in the Astropy-affiliated package dust_extinction, with the F19 Milky Way $R(V)$ dependent extinction (Fitzpatrick et al. 2019) using the standard value $R v=3.1$ and total extinction: $E_{B-V}=0.3$ (de Ruyter et al. 2005).

We made use of the astropy.modeling.blackbody routine (Astropy Collaboration et al. 2013) to depict scaled blackbody components on the SED (these are not fitted). We include two blackbody components with temperatures of 5000 and $1100 \mathrm{~K}$ to represent the stellar atmosphere (Bódi \& Kiss 2019) and the inner-disk edge (see Section 4.4), respectively. We also include a modified blackbody (i.e., "graybody"; Casey 2012) component with a mean temperature of $350 \mathrm{~K}$ to represent the 
dust in the extended disk. Finally, we added a $3.5 \mathrm{MK}$ thermal blackbody for the X-ray spectrum. ${ }^{19}$ Figure 5 shows the entire SED model as a black solid line, with the individual components depicted as light-gray dashed lines.

We integrated the $5000 \mathrm{~K}$ blackbody model (between $\sim 0.2$ and $2 \mu \mathrm{m}$ ), which gave a (dereddened) bolometric flux of $\mathrm{F}_{\mathrm{bol}} \approx 1.44 \times 10^{-7} \mathrm{erg} \mathrm{s}^{-1} \mathrm{~cm}^{-2}$, corresponding to $L$ $\sim 5556 L_{\odot}$ and yielding a physical radius of $R_{*} \sim 100 R_{\odot}$.

We tested how an additional blackbody component with the properties of the putative A-type companion star $(9800 \mathrm{~K}$ and $55 L_{\odot}$ ) would influence our SED model and found that such a companion would remain undetectable in the SED (even at $\mathrm{RVb}$ minimum) owing to the brightness of the post-AGB component (see Section 5.3).

Assuming that the dust is optically thin at $\lambda=870 \mu \mathrm{m}$, we used the following expression (e.g., Hildebrand 1983) to estimate the mass of the dust in the disk:

$$
M_{d}=\frac{F_{\lambda} D^{2}}{\kappa_{\lambda} B_{\lambda}\left(T_{d}\right)},
$$

where $B_{\lambda}\left(T_{\mathrm{d}}\right)$ is the blackbody intensity at the Rayleigh-Jeans limit in the form of $\frac{2 k_{S B} T}{\lambda^{2}}$. Using a mean dust temperature $T_{\mathrm{d}}=350 \mathrm{~K}$, our observed SMA flux $F_{870}=173.3 \mathrm{mJy}$, $D=1.1 \mathrm{kpc}$ (Bódi \& Kiss 2019), and a dust opacity of $\kappa_{\lambda}=2.0 \mathrm{~cm}^{2} \mathrm{~g}^{-1}$ at $870 \mu \mathrm{m}$ (Ladjal et al. 2010), we estimate a total dust mass of $M_{\mathrm{d}} \sim 4 \times 10^{-4} M_{\odot}$. For a typically assumed gas-to-dust ratio of 200 (e.g., Groenewegen et al. 2007, 2009) the total dust mass suggests a gas mass of $\sim 8 \times 10^{-2} M_{\odot}$; however, this gas mass is much larger than the constraint of the molecular mass estimated by Bujarrabal et al. (2013). The lack of molecular gas may be related to high-energy radiation that has been dissociating molecules present in the system that would support the larger distance to U Mon. Since the primary star is not hot enough, the presence of a hotter companion or interstellar UV field could photodissociate molecules in the inner and outer disk regions (Bujarrabal et al. 2013).

\subsection{Properties of the Circumbinary Disk's Inner Edge}

The radius of the near-IR emission, where dust can be sublimated by stellar radiation, can also set the physical radius of the inner boundary of the disk (Dullemond et al. 2001; Monnier \& Millan-Gabet 2002; Hillen et al. 2017; Kluska et al. 2019; Lazareff et al. 2017). Using the following luminosity - radius relation (e.g., Lazareff et al. 2017), we estimated the inner-rim radius $\left(R_{\text {rim }}\right)$ of the CBD for $\mathrm{U}$ Mon:

$$
R_{\text {rim }}=\frac{1}{2}\left(\frac{C_{\mathrm{bw}}}{\epsilon}\right)^{1 / 2}\left(\frac{L_{\star}}{4 \pi \sigma T_{\mathrm{sub}}^{4}}\right)^{1 / 2}
$$

where we adopted $L_{\star}=5480 L_{\odot}$ (Bódi \& Kiss 2019) as the stellar luminosity. $C_{\mathrm{bw}}$ is the back-warming coefficient of the inner-disk edge that ranges between $\sim 1$ and 4 , where $C_{\mathrm{bw}}=1$ provides a lower limit on $R_{\text {rim }}$ and $C_{\mathrm{bw}}=4$ provides an upper limit on $R_{\text {rim }}$ (Monnier et al. 2005; Kama et al. 2009). The cooling efficiency of the dust grains, defined by $\epsilon=\kappa\left(T_{\text {dust }}\right) / \kappa\left(T_{\star}\right)$, is a ratio between

\footnotetext{
19 The blackbody model is only shown for display purposes, the unfolded spectrum has a strong dependence on the best-fit $10 \mathrm{MK}$ plasma model, and a blackbody model does not provide a suitable fit to the observed X-ray spectrum.
}

Table 3

Results of Model Fits to Visibilities of U Mon Measured with the SMA

\begin{tabular}{lcccc}
\hline \hline Band & Model & $\begin{array}{c}\text { Major } \\
\text { (mas) }\end{array}$ & Minor/Major & $\begin{array}{c}\text { P.A. } \\
(\mathrm{deg})\end{array}$ \\
\hline $1.3 \mathrm{~mm}$ & Gaussian & $299 \pm 11$ & $0.73 \pm 0.04$ & $83 \pm 5$ \\
$1.3 \mathrm{~mm}$ & Unif. disk & $483 \pm 24$ & $0.76 \pm 0.05$ & $84 \pm 8$ \\
$0.8 \mathrm{~mm}$ & Gaussian & $244 \pm 6$ & $0.86 \pm 0.03$ & $55 \pm 7$ \\
$0.8 \mathrm{~mm}$ & Unif. disk & $482 \pm 64$ & $1.0 \pm 0.2$ & $\cdots$ \\
\hline
\end{tabular}

Note. For Gaussian models, FWHM is given as the major-axis size.

the Planck mean opacity $(\kappa)$ of the dust species at its own temperatures and that at the stellar temperature, where $\epsilon \leqslant 1$, and generally increases with grain size (Kama et al. 2009). For the dust in the CBD, we assume $\epsilon \sim 1$. Typically, for oxygen-rich dust species ([C/O] of 0.8 for UMon), the sublimation temperature ( $T_{\text {sub }}$ ) is $\sim 1100 \mathrm{~K}$ (Bladh et al. 2013). We note that Kluska et al. (2019) suggest a higher inner-dust temperature $(\sim 2600 \mathrm{~K})$ for U Mon but only reach a moderate fit with their most complex model, whereas most of their sample of post-AGB binaries have near-IR circumstellar emission sublimation temperatures lower than $1200 \mathrm{~K}$. Finally, the range between $C_{\mathrm{bw}}=1$ and $C_{\mathrm{bw}}=4$ gives an $R_{\text {rim }}$ between $\sim 4.5$ and 9.0 au, respectively.

\subsection{Submillimeter Emission from UMon}

The SMA observations in the combined array configurations and uniform weighting of visibilities resulted in continuum maps with synthesized beam sizes of 0 ." $69 \times 0$ ". 37 at $1.3 \mathrm{~mm}$ and 0 .' $39 \times 0$.' 24 at $0.87 \mathrm{~mm}$, respectively. Simple fits of elliptical Gaussians to the map at $0.87 \mathrm{~mm}$ give a source size of $(0$ ". $89 \pm 0$ ". 34) $\times(0$ ". $20 \pm 0$ ". 39$)$ and an orientation of the longer axis at a position angle (P.A.) of $55^{\circ} \pm 24^{\circ}$. The map at $1.3 \mathrm{~mm}$ indicates that the source size is much smaller than the beam, i.e., with FWHM $\lesssim 0$ ". 55 . To better constrain the size and make best use of the data, we directly tried to fit a model source to the calibrated visibilities. Best solutions for an elliptical Gaussian and an elliptical uniform disk were found in a CASA task uvmodelfit and are presented in Table 3.

The model fits indicate a nearly circular source with a Gaussian FWHM smaller than about 500 mas, which corresponds to a disk diameter $\lesssim 550$ au at the nominal distance of $1.1 \mathrm{kpc}$. A denser $u v$ coverage of observations at longer baselines would be necessary to better constrain the size of the submillimeter source.

The SMA fluxes are consistent with earlier observations in the nearby bands and represent the coolest component of U Mon's SED, which is consistent with a dusty source of $T_{d}=350 \mathrm{~K}$ (see Section 4.3).

\subsection{X-Ray and UV Emission from U Mon}

This is the first X-ray detection of an RV Tauri variable by any X-ray telescope. The X-rays are consistent with the location of $U$ Mon within the pointing uncertainties and the fairly broad point-spread function of the XMM EPIC X-ray detectors $\left(\mathrm{FWHM} \sim 12^{\prime \prime}\right)$. There is an additional but fainter source near the position of U Mon with a separation of $\sim 20^{\prime \prime}$ (see Figure 4). Given the relative brightnesses of the two sources and the radius used to extract the X-ray products, contamination by the nearby source is minimal. 
Table 4

X-ray Spectral Fit Results for U Mon

\begin{tabular}{lc}
\hline \hline Quantity & Value \\
\hline$N_{\mathrm{H}}\left(10^{21} \mathrm{~cm}^{2}\right)$ & $1.2_{-0.5}^{+0.6}$ \\
$T_{\mathrm{X}}\left(10^{6} \mathrm{~K}\right)$ & $12_{-2}^{+1}$ \\
Oxygen abundance ${ }^{\mathrm{a}}$ & $3.4_{-1.6}^{+3.0}$ \\
Iron abundance & 0.16 \\
Model normalization $\left(10^{-5}\right)$ & $2.1_{-0.3}^{+0.3}$ \\
$F_{\mathrm{X}, \mathrm{abs}} \mathrm{b}\left(10^{-14} \mathrm{erg} \mathrm{cm}^{-2} \mathrm{~s}^{-1}\right)$ & $2.2_{-0.4}^{+0.4}$ \\
$F_{\mathrm{X}, \text { unabs }}\left(10^{-14} \mathrm{erg} \mathrm{cm}^{-2} \mathrm{~s}^{-1}\right)$ & $3.4_{-0.6}^{+0.6}$ \\
EM $\left(10^{53} \mathrm{~cm}^{-3}\right)$ & $3_{-1}^{+1}$ \\
$L_{\mathrm{X}}\left(10^{30} \mathrm{erg} \mathrm{s}^{-1}\right)$ & $5_{-1}^{+1}$ \\
\hline
\end{tabular}

Notes.

a Abundances are number relative to solar values from Anders \& Grevesse (1989). Iron abundance has been fixed to the $[\mathrm{Fe} / \mathrm{H}]$ value of Giridhar et al. (2000) after conversion to the number relative value.

${ }^{\mathrm{b}}$ Model-derived fluxes have been corrected for the encircled energy fraction of the extraction region.

The spectra were modeled using an absorbed (tbabs; Wilms et al. 2000), optically thin plasma model with variable abundances (vapec; Smith et al. 2001; Foster et al. 2012). Model fitting was performed with XSPEC (Arnaud 1996). No acceptable fit could be found with solar abundances, but adequate fits were found when Fe was allowed to vary. Given the limited energy resolution and moderately low count rate of the CCD spectrum, we could not obtain meaningful constraints on both the plasma properties (temperature and normalization) and the important elemental abundances in the energy range of the detected emission ( $\mathrm{C}, \mathrm{N}, \mathrm{O}, \mathrm{Ne}, \mathrm{Mg}$, and $\mathrm{Fe}$ ). Instead, we fixed the $\mathrm{Fe}$ abundance to a previously reported value of $[\mathrm{Fe} / \mathrm{H}]=-0.79$ and the C-to-O ratio to 0.8 (Giridhar et al. 2000). The $O$ abundance was allowed to vary along with the overlying absorption, plasma temperature, and plasma model normalization. The best-fit parameters are presented in Table 4 along with the absorbed and intrinsic X-ray fluxes and source luminosity for a distance of $1.1 \mathrm{kpc}$ (Bódi \& Kiss 2019). The best-fit spectral model is displayed with the X-ray spectra in Figure 8, and the unfolded spectrum is presented in the multiwavelength SED in Figure 5; confidence level contours are shown in Figure 9.

Our analysis of the X-ray observations suggests that the emission is consistent with an iron-deficient hot $(\sim 10 \mathrm{MK})$ plasma with moderate absorption. The absorption reported in Table 4 is consistent with extinction to $\mathrm{U}$ Mon $\left(E_{B-V} \sim\right.$ 0.3 mag; de Ruyter et al. 2005). The X-ray light curve reveals no evidence of flaring activity at the $3 \sigma$ level; however, the light curves required $10 \mathrm{ks}$ temporal bins to net sufficient signal-to-noise ratio. Hence, we cannot evaluate activity on shorter timescales. We discuss the origin of the X-ray emission in further detail in Section 5.3.

The XMM Optical Monitor performed numerous exposures in three UV filters: UVW1, UVM2, and UVW2 (with effective wavelength of $0.291,0.231$, and $0.212 \mu \mathrm{m}$, respectively). U Mon is detected in all three bands but saturated in UVW1. The UVM2 and UVW2 measurements are included in the multiwavelength SED in Figure 5 and in Table 2. The UV light curves show variability consistent with Poisson noise. Given the short time frame for the UV observations, we are not very sensitive to variations or trends related to the pulsation period or the long-period variation.

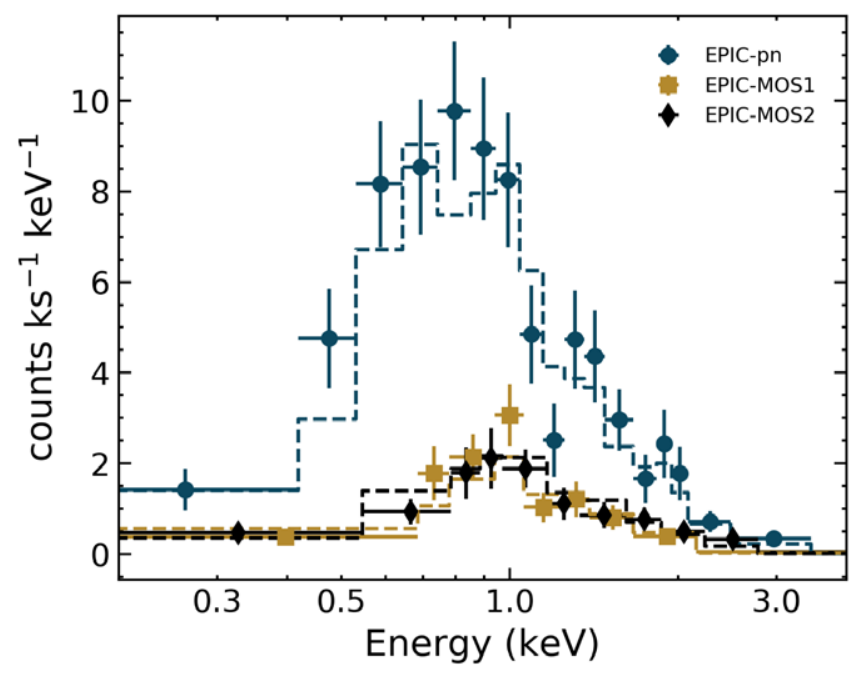

Figure 8. U Mon's X-ray spectra and best-fit spectral model (see Table 4).

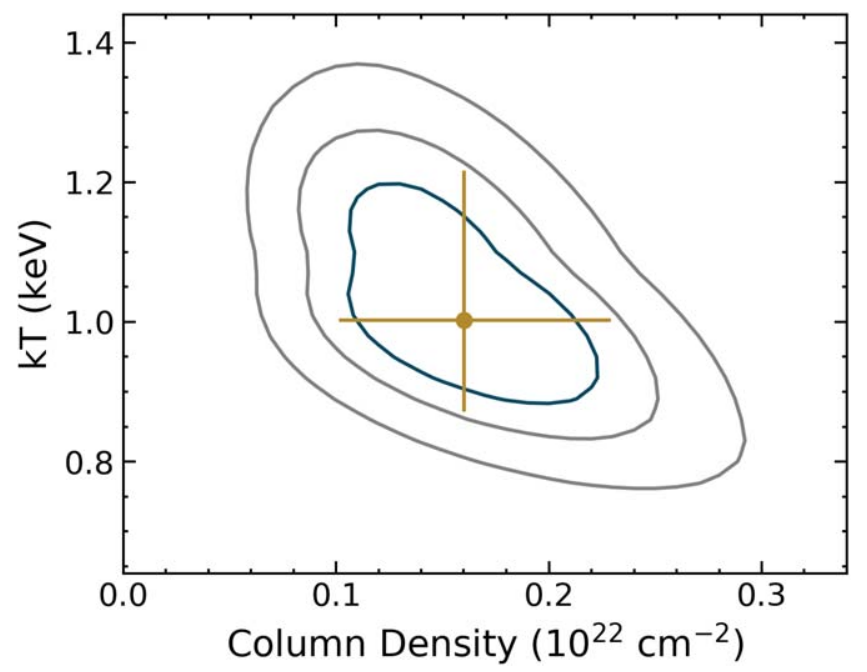

Figure 9. Best-fit confidence map for the plasma temperature $(k T)$ and column density $\left(N_{\mathrm{H}}\right)$ of the X-ray spectral fit. The best-fit solution is depicted with a $98 \%$ confidence range, and $68 \%, 90 \%$, and $98 \%$ confidence levels are depicted.

\section{Discussion}

\subsection{A 60 yr Trend in the Light Curve}

U Mon's RVb behavior has been observed since before the 1900s, giving a window into the behavior of the CBD. Even with the lack of uniform monitoring in combination with various physical processes present (e.g., pulsations), an even slower trend in the light curve is still detected in the AAVSO + DASCH light curve.

In particular, three RVb cycles between 1931 and 1951 have significantly more scatter, compared to the overall light curve. They resemble three cycles between 1991 and 2011 (see Figure 7). The disk-shadowing interpretation can be a possible explanation for the disappearance of the long-term variation or the lack thereof. This phenomenon can represent times when the RVb minima have become shallower and shorter, perhaps indicating some kind of precession of the binary or of disk-structure inhomogeneity at the inner-disk edge caused by binary-disk interaction (e.g., Kluska et al. 2018; Oomen et al. 2020).

Furthermore, the light curve hints of a different feature of two even deeper RVb minima that occur around 1925 and more 
clearly around 1985 (phase $\sim 34$ yr in Figure 7) with the same time separation of $\sim 60.4 \mathrm{yr}$. A warp or a dense feature at the inner edge of the disk orbiting around it would only take about $6 \mathrm{yr}$ (2190 days), which is comparable to the orbital period. If a warp or dense feature is farther out in a Keplerian orbit, it would have to be at a distance of 25 au to block out more of the post-AGB so that the RVb minimum becomes deeper every $60.4 \mathrm{yr}$.

As more photometric monitoring and interferometric imaging become available for post-AGB binaries, the detection of CBD warps or features may give rise to future investigations on possible second-generation planet formation such as in studies around other evolved stars (e.g., Hardy et al. 2016; Homan et al. 2018).

\subsection{Circumbinary Disk Interaction}

Pollard \& Cottrell (1995) and Pollard et al. (2006) found enhanced $\mathrm{H} \alpha$ emission in $\mathrm{U}$ Mon during the $\mathrm{RVb}$ minima. This phenomenon is reminiscent of one observed in other binary star systems with eccentric orbits within CBDs, in which matter is pulled from the inner edge of the disk and onto the stars at apastron (e.g., Artymowicz \& Lubow 1996; Basri et al. 1997). Based on the periastron time occurring at $\mathrm{RVb}$ maximum, we experiment with the idea that the relevant time to explain the interaction that produces $\mathrm{H} \alpha$ enhancement is not at periastron but rather the time when the two stars are farthest apart (apastron), which would be when the stars would presumably be most likely to interact with the inner edge of the CBD.

Based on our orbital fit for the binary, the argument of periastron $\left(\omega=95^{\circ} \pm 7^{\circ}\right)$ means that the longest dimension of the ellipse is oriented parallel to our line of sight. In other words, when the two stars are farthest apart, they are also along our line of sight during apastron, which happens during $\mathrm{RVb}$ minima. This scenario requires that the post-AGB be mostly hidden by the CBD while the companion travels in the background at an elevated angle crossing our light of sight and possibly interacting with the inner edge of the CBD, causing the enhanced $\mathrm{H} \alpha$ emission observed by Pollard et al. (2006) during $\mathrm{RVb}$ minima. This is also shown from the radial velocities in Figure 1, which measure the motion of the postAGB star. The orbital phase of zero is at periastron (which corresponds to $\mathrm{RVb}$ maximum).

The nature of the CBD-binary interaction is not yet clear in post-AGB binaries; however, processes such as photospheric chemical depletion show that it is crucial. Interestingly, U Mon's photosphere does not show signs of depletion (Giridhar et al. 2005; Gezer et al. 2015); this could be because depletion is mostly observed in stars hotter than $5000 \mathrm{~K}$ (Venn et al. 2014). It is also possible that the depletion process could be interrupted by wind Roche lobe overflow onto the companion (Mohamed \& Podsiadlowski 2007).

The recent study by Oomen et al. (2020) investigated a few disk-binary interaction mechanisms for post-AGB binaries with orbital periods of 100-2500 days and eccentricities $\gtrsim 0.3$. They concluded that disk-binary interactions are unlikely to pump the eccentricity to the observed values within the evolutionary timescales for the stars they sampled. U Mon is not very eccentric $(e=0.31)$; this may suggest a weak disk - binary interaction.

However, the Kluska et al. (2019) geometric image reconstruction model of U Mon's visibility profile resulted in a fit that displayed strong azimuthal modulation, showing that this system likely has a very complex inner-disk morphology. Additionally, they showed that U Mon has a relatively large mid-IR-to-near-IR size ratio. The authors note that it is possible that the model is not able to reproduce all the complexities, which could have an effect on their derived size of the CBD's inner rim; thus, further observations will bring stronger constraints. Such complexities have been seen in some YSO CBD systems, which can be regarded as scaled-down versions of the CBDs around post-AGB binaries (e.g., Hillen et al. 2017). For example, in the CBD of the young binary GW Ori, imaging observations by Kraus et al. (2020) find evidence for an inner ring and a large warp in the outer part of the disk. This phenomenon occurs when the outer disk is misaligned with the orbital plane, so it wraps and breaks into precessing rings, which may provide a mechanism for planet formation.

Bollen et al. (2019) provide a detailed investigation on the possibilities for jets in explaining the $\mathrm{H} \alpha$ emission feature observed in post-AGB systems. In their model, the $\mathrm{H} \alpha$ feature is superimposed on the photospheric absorption from the postAGB star and is present throughout the entire orbit. To establish whether such a jet scenario may apply to UMon as well would require phase-resolved, high-resolution spectroscopy of the $\mathrm{H} \alpha$ feature.

If, instead, there is a stream outflow coming off the CBD onto the companion, always traveling around the companion and opposite of the post-AGB primary, the $\mathrm{H} \alpha$ enhancement would have a timescale near the binary orbital period. The stream of material would be at a focus point where it would be enhanced around apastron because that would be the shortest distance the CBD material would flow onto the companion. The stability for the shortest orbit of material around a binary should be at least $\sim 3-4$ times the binary separation (Holman \& Wiegert 1999). However, it is possible that the inner edge of RV Tauri disks may not be in long-term stable orbits. Artymowicz \& Lubow (1996) showed hydrodynamical models for unstable inner-disk rims, so it is possible that material may be stripped by the binary, in particular the companion. This scenario may also explain the X-rays observed in U Mon.

\subsection{Nature and Origin of X-Ray Emission from UMon}

As a binary system with a CBD, there are a number of processes that can generate the detected X-ray emission. We consider processes from U Mon, the companion, and interactions between the components of the system including the CBD.

If the X-ray emission originates from $\mathrm{U}$ Mon, the X-ray luminosity corresponds to an $L_{\mathrm{X}} / L_{\mathrm{bol}}=10^{-7}$. Generally, such a ratio is consistent with the $\mathrm{X}$-ray emission that arises from shocks caused by variable winds in $\mathrm{O}$ and $\mathrm{B}$ stars; however, the $\mathrm{X}$-ray emission from $\mathrm{U}$ Mon does not quite follow the general trend with $L_{\text {bol }}$ seen for B stars in the Carina Nebula, which would predict an X-ray luminosity up to an order of magnitude higher (e.g., Nazé et al. 2011). The plasma temperature ( $>10$ $\mathrm{MK}$ ) is consistent with hot components found in the Carina $\mathrm{O}$ and B stars; however, no fast outflows have been measured from $\mathrm{U}$ Mon. Indeed, the large radius of U Mon suggests an escape velocity $<100 \mathrm{~km} \mathrm{~s}^{-1}$, whereas the plasma temperature requires velocities $>800 \mathrm{~km} \mathrm{~s}^{-1}$ for a strong shock. Such velocities have not been measured from $U$ Mon and are unlikely to be produced by any pulsation-induced shocks in the atmosphere of U Mon (Fokin 1994), unlike that suggested for $\mathrm{X}$-ray emission from the Cepheid star $\delta$ Cep (Engle et al. 2017). 
Gillet et al. (1990) measured shock amplitudes from spectroscopy for the RV Tauri variables R Sct and AC Her that did not exceed $\sim 40 \mathrm{~km} \mathrm{~s}^{-1}$.

We note that Moschou et al. (2020) recently investigated a mechanism to produce phase-dependent shocked gas X-ray emission in the pulsating atmosphere of classical Cepheids. This mechanism requires the presence of solar-like coronal plasma into which the phase-dependent shocks are driven (Moschou et al. 2020). The detected X-ray emission from U Mon is hotter than that studied by Moschou et al. (2020), and we lack adequate phase coverage to establish the presence of such shocks from U Mon, as well as the necessary signal-tonoise ratio in our XMM X-ray light curve to establish the presence of such shocks from U Mon. A solar-like coronal plasma is an intriguing notion given the detection of a magnetic field (very likely dynamo generated) at the surface of this cool post-AGB star, but there is, so far, no information on any related activity such as flares, stellar spots, or dynamo variability that would be linked to X-ray emission. Furthermore, the ability to sustain a hot stellar corona on such a large evolved star is unclear (Sahai et al. 2015).

Accretion, from a compact disk or infalling material from the large CBD (as discussed in Section 5.2), might create shocks that can heat material up to X-ray-emitting temperatures. However, given the stellar properties of U Mon in Table 1, it is unlikely that infalling material will reach sufficiently high velocities $\left(<100 \mathrm{~km} \mathrm{~s}^{-1}\right.$ at the surface of $U$ Mon $)$. If the companion star has a radius $\leqslant 2 R_{\odot}$, which would make the companion consistent with an A-type main-sequence star, infalling material, from the CBD or donated by UMon via wind Roche lobe overflow (Mohamed \& Podsiadlowski 2007), can reach speeds in excess of $800 \mathrm{~km} \mathrm{~s}^{-1}$. Such infalling material can either slam into the surface of the star along magnetic fields or form into an accretion disk around the companion.

An accretion disk forming around the companion could then generate outflows as seen in other post-AGB systems (Gorlova et al. 2012, 2015); however, such outflows have yet to be observed from the U Mon system. If such an outflow exists, its velocity could be sufficient to generate shocks capable of explaining the detected X-ray emission. Detailed monitoring of the $\mathrm{H} \alpha$ emission from U Mon, like that performed of similar systems by Gorlova et al. (2012, 2013), could help establish the presence of accretion-disk-driven outflows.

If the binary companion is the source of X-ray emission, in addition to accretion, coronal activity and radiative stellar winds can produce X-ray emission similar to that given in Table 4. However, at $\sim 2.2 M_{\odot}$ (Table 1 ), a main-sequence companion would be consistent with an early-type A star and unable to support magnetic activity to produce coronal X-ray emission (Stelzer et al. 2006). Furthermore, the bolometric luminosity of such a main-sequence companion is 100 times fainter than $\mathrm{U}$ Mon, leading to an $L_{\mathrm{X}} / L_{\mathrm{bol}}$ of $10^{-5}$ for the companion, which is an unusually high fraction for an A-type star. Such a high fraction of X-ray emission and a hightemperature plasma are comparable to what is seen from Herbig $\mathrm{Ae} / \mathrm{Be}$ stars, which are intermediate-mass pre-mainsequence stars embedded in dusty disks (Stelzer et al. 2006, 2008). In the case of Herbig stars, the high plasma temperature suggests that radiative winds are not the emission mechanism, and higher-order binary components, namely, unseen rapidly rotating late-type companions to the $\mathrm{A}$ star in a
Herbig system, have been suggested as a potential origin for their X-ray emission (Stelzer et al. 2006). Since we have not observed the companion and only have a modest constraint on its mass $\left(2.2_{-0.75}^{+1.0} M_{\odot}\right.$; see Table 1$)$, we cannot exclude the remote possibility that the companion is a compact binary. In such a scenario, the detected X-ray emission would be consistent with enhanced coronal activity from rapidly rotating late-type stars in a close binary system.

We have highlighted a number of potential origins for the X-ray emission from the UMon system, each with strong implications for the nature and evolution of the system. Additional supporting information, such as further constraints on the companion properties and monitoring of the $\mathrm{H} \alpha$, like that reported in Bollen et al. (2019), and additional observations of the X-ray emission through the phases of the U Mon pulsation and orbit, is essential to better understand the origin and influence of the X-ray emission.

\section{Conclusions}

We have conducted the most comprehensive characterization yet of an RVb system, U Mon, with observations spanning the largest range of wavelengths (X-ray to millimeter) and the longest range of time $(\sim 130 \mathrm{yr})$.

U Mon, one of the brightest RV Tauri variables of its class, is shown here to be a binary system comprising a $2.07 M_{\odot}$ postAGB star that has lost mass relative to a more massive unevolved $2.2 M_{\odot}$ A-star companion, and is surrounded by a large $\mathrm{CBD}$. We obtained new orbital parameters within the errors of the values found by Oomen et al. (2018), by fixing the orbital period as the photometric $\mathrm{RVb}$ period. The new periastron value, $T_{0}=2,452,203$ days, reveals that the apastron times align with RVb minima (Figure 1). Additionally, based on our orbital fit, the argument of periastron $\left(\omega=95^{\circ}\right)$ translates to the long axis of the orbit being roughly aligned along our line of sight. This supports the argument that apastron occurs when the post-AGB star is occulted by the near side of the disk at $\mathrm{RVb}$ minimum while the A-type star companion is most revealed at the far side of the inner hole in the CBD. Moreover, we found that the binary's semimajor-axis separation is $5.78 \mathrm{au}$, which at apastron is comparable to the size range of the inner-disk hole radius $(\sim 4.5-9.0 \mathrm{au})$.

U Mon shows several interesting features, some not yet seen in any other post-AGB binary, such as a magnetic field and most recently X-rays. We found a $\sim 10 \mathrm{MK}$ plasma model that is consistent with the X-ray spectra and considered origins from U Mon and/or its companion. We suggest that at apastron the companion may be close enough to strip material from the CBD, creating the X-ray emission observed. This interpretation may agree with the complex morphology found in the interferometric data set for U Mon by Kluska et al. (2019).

The occurrence of the enhanced $\mathrm{H} \alpha$ at RVb minima (e.g., Pollard et al. 2006) also seems to correspond to when the A-type star companion is most revealed at apastron, implying that since the post-AGB star is shadowed by the CBD at this phase, the enhanced $\mathrm{H} \alpha$ must not be coming from the postAGB star but rather from the environment around the A-type companion, likely an accretion disk (e.g., Bollen et al. 2019). Recent work has shown that the $\mathrm{H} \alpha$ variation is due to a bipolar jet from the circumcompanion accretion disks in other postAGB binaries. More time series of optical spectra are needed to investigate the $\mathrm{H} \alpha$ variability in U Mon. 
We tested whether additional blackbody components with properties of the A-type star companion would influence U Mon's SED but found that such a companion seems to only be detected in $\mathrm{H} \alpha$ (and possibly X-rays), even at $\mathrm{RVb}$ minimum, when it is most revealed in its orbit. The X-ray through millimeter emission, however, is consistent with components that include a hot thermal plasma, the stellar photosphere of the post-AGB, and the inner- and extended-disk emission. The scaling of the modified blackbody for the dust emission includes parameters such as opacity and dust grain sizes that we do not explore in this paper. However, U Mon's new SMA observation fluxes are in agreement with the slope of the Rayleigh-Jeans form of the Planck function, between the other 60-3000 $\mu \mathrm{m}$ flux measurements. Such slopes are consistent with the presence of a composition of large dust grains (radius $\gtrsim 0.1 \mathrm{~mm}$; de Ruyter et al. 2005). Due to the short SMA observations, we are only able to estimate upper limits on U Mon's disk diameter size $\lesssim 550$ au (see Table 3 ).

Nevertheless, with the AAVSO+DASCH light curve, which spans more than $130 \mathrm{yr}$, we find evidence for a $60.4 \mathrm{yr}$ cycle that could be due to some structure in the disk at a separation of $\sim 25$ au that temporarily allows the post-AGB star to avoid being occulted at apastron $/ \mathrm{RVb}$ minimum. Accordingly, the 60.4 yr cycle also produces an especially deeper RVb minimum (phase $\sim 54$ yr in Figure 7-two cycles before the "disappearance" of the RVb minima). Presumably this can be a corresponding feature in the disk that causes an exceptionally large occultation of the post-AGB star. More radio observations at longer baselines would constrain the size of the disk, as well as resolve features we see in U Mon's light curve.

Furthermore, the detection of X-rays from the U Mon system has opened up new possibilities; do all RV Tauri variables exhibit X-ray emission? In order to answer this question, $\mathrm{X}$-rays will be an important factor to consider in forthcoming RV Tauri studies to enable constraints on their evolution (Graber \& Montez 2021).

Most importantly, U Mon now becomes an archetype for the study of binary post-AGB stellar environments that represent an important evolutionary phase, which either sets the stage for sculpting the morphology and evolution of planetary nebulae or may represent systems that never become planetary nebulae at all, as the disk and stellar companion arrest its development.

We acknowledge the very helpful review of the anonymous referee, which substantively improved the paper. We acknowledge the excellent work of NASA's Goddard Space Flight Center's Astrophysics Science Division Communications Team in the production of a NASA news feature for this research. L.D.V. and K.G.S acknowledge the support of the NASA MUREP Harriett G. Jenkins Pre-doctoral Fellowship grant Nos. NNX15AU33H and 80NSSC19K1292. R.M. and L.D.V. acknowledge the federal support from the Latino Initiatives Pool, administered by the Smithsonian Latino Center. This research has made use of XMM-Newton, an ESA Science Mission with instruments and contributions directly funded by ESA Member States and the USA (NASA). This research has made use of the Submillimeter Array, a joint project between the Smithsonian Astrophysical Observatory and the Academia Sinica Institute of Astronomy and Astrophysics, and is funded by the Smithsonian Institution and the Academia Sinica. We acknowledge with thanks the variable star observations from the AAVSO International Database contributed by observers worldwide and used in this research. This research has made use of the DASCH project at Harvard, which is grateful for partial support from NSF grants AST-0407380, AST-0909073, and AST-1313370. This research has made use of the SIMBAD database, operated at CDS, Strasbourg, France, and of the VizieR catalog access tool, CDS, Strasbourg, France (DOI: $10.26093 / \mathrm{cds} /$ vizier). The original description of the VizieR service was published in 2000 (Ochsenbein et al. 2000).

Facilities: AAVSO, DASCH, SMA, XMM-Newton.

Software: Astropy (Astropy Collaboration et al. 2013), CASA (McMullin et al. 2007), MIR (http://github.com/qimolecules/sma-mir), PHOEBE (Prša et al. 2016), XSPEC (Arnaud 1996).

\section{ORCID iDs}

Laura D. Vega (iD https://orcid.org/0000-0002-5928-2685 Keivan G. Stassun (ib https://orcid.org/0000-0002-3481-9052 Rodolfo Montez, Jr. (D) https://orcid.org/0000-0002-6752-2909 Tomasz Kamiński (iD https://orcid.org/0000-0002-1113-9150 Laurence Sabin (iD https://orcid.org/0000-0003-0242-0044 Eric M. Schlegel (ib https://orcid.org/0000-0002-4162-8190 Wouter H. T. Vlemmings (ib https://orcid.org/0000-00022700-9916

Joel H. Kastner (iD https://orcid.org/0000-0002-3138-8250

Sofia Ramstedt (iD https://orcid.org/0000-0001-5177-6202

Patricia T. Boyd (iD https://orcid.org/0000-0003-0442-4284

\section{References}

Alcock, C., Allsman, R. A., Alves, D. R., et al. 1998, AJ, 115, 1921

Anders, E., \& Grevesse, N. 1989, GeCoA, 53, 197

Arnaud, K. A. 1996, in ASP Conf. Ser. 101, Astronomical Data Analysis Software and Systems V, ed. G. H. Jacoby \& J. Barnes (San Francisco, CA: ASP), 17

Artymowicz, P., \& Lubow, S. H. 1996, ApJL, 467, L77

Astropy Collaboration, Robitaille, T. P., Tollerud, E. J., et al. 2013, A\&A, 558, A33

Basri, G., Johns-Krull, C. M., \& Mathieu, R. D. 1997, AJ, 114, 781

Belokurov, V., Penoyre, Z., Oh, S., et al. 2020, MNRAS, 496, 1922

Bladh, S., Höfner, S., Nowotny, W., Aringer, B., \& Eriksson, K. 2013, A\&A, 553, A20

Bódi, A., \& Kiss, L. L. 2019, ApJ, 872, 60

Bollen, D., Kamath, D., De Marco, O., Van Winckel, H., \& Wardle, M. 2020, A\&A, 641, A175

Bollen, D., Kamath, D., Van Winckel, H., \& De Marco, O. 2019, A\&A, 631, A53

Bollen, D., Van Winckel, H., \& Kamath, D. 2017, A\&A, 607, A60

Bono, G., Castellani, V., \& Marconi, M. 2000, ApJ, 529, 293

Bujarrabal, V., Alcolea, J., Van Winckel, H., Santander-García, M., \& Castro-Carrizo, A. 2013, A\&A, 557, A104

Bujarrabal, V., Castro-Carrizo, A., Alcolea, J., \& Neri, R. 2005, A\&A, 441, 1031

Bujarrabal, V., Castro-Carrizo, A., Van Winckel, H., et al. 2018, A\&A, 614, A58

Casey, C. M. 2012, MNRAS, 425, 3094

de Ruyter, S., Van Winckel, H., Dominik, C., Waters, L. B. F. M., \& Dejonghe, H. 2005, A\&A, 435, 161

de Ruyter, S., Van Winckel, H., Maas, T., et al. 2006, A\&A, 448, 641

Deroo, P., Van Winckel, H., Min, M., et al. 2006, A\&A, 450, 181

Dullemond, C. P., Dominik, C., \& Natta, A. 2001, ApJ, 560, 957

Engle, S. G., Guinan, E. F., Harper, G. M., et al. 2017, ApJ, 838, 67

Evans, T. L. 1985, MNRAS, 217, 493

Fitzpatrick, E. L., Massa, D., Gordon, K. D., Bohlin, R., \& Clayton, G. C. 2019, ApJ, 886, 108

Fokin, A. B. 1994, A\&A, 292, 133

Foster, A. R., Ji, L., Smith, R. K., \& Brickhouse, N. S. 2012, ApJ, 756, 128

Gaia Collaboration, Brown, A. G. A., Vallenari, A., et al. 2018, A\&A, 616, A1 Gandhi, P., Buckley, D. A. H., Charles, P., et al. 2020, arXiv:2009.07277 Gehrz, R. D. 1972, ApJ, 178, 715

Gezer, I., Van Winckel, H., Bozkurt, Z., et al. 2015, MNRAS, 453, 133 
Gielen, C., Bouwman, J., Van Winckel, H., et al. 2011, A\&A, 533, A99 Gillet, D., Burki, G., \& Duquennoy, A. 1990, A\&A, 237, 159

Giridhar, S., Lambert, D. L., \& Gonzalez, G. 2000, ApJ, 531, 521

Giridhar, S., Lambert, D. L., Reddy, B. E., Gonzalez, G., \& Yong, D. 2005, ApJ, 627, 432

Gorlova, N., Van Winckel, H., Gielen, C., et al. 2012, A\&A, 542, A27

Gorlova, N., Van Winckel, H., Ikonnikova, N. P., et al. 2015, MNRAS, 451, 2462

Gorlova, N., Van Winckel, H., Vos, J., et al. 2013, in EAS Publications Series, Vol. 64, Setting a New Standard in the Analysis of Binary Stars, ed. K. Pavlovski, A. Tkachenko, \& G. Torres (Paris: EDP Sciences), 163

Gorlova, N., Van Winckel, H., Vos, J., et al. 2014, arXiv:1403.2287

Graber, S., \& Montez, R. 2021, BAAS, 53, 528

Grindlay, J., Tang, S., Simcoe, R., et al. 2009, in ASP Conf. Ser. 410, Preserving Astronomy's Photographic Legacy: Current State and the Future of North American Astronomical Plates, ed. W. Osborn \& L. Robbins (San Francisco, CA: ASP), 101

Groenewegen, M. A. T., \& Jurkovic, M. I. 2017, A\&A, 604, A29

Groenewegen, M. A. T., Sloan, G. C., Soszyński, I., \& Petersen, E. A. 2009, A\&A, 506, 1277

Groenewegen, M. A. T., Wood, P. R., Sloan, G. C., et al. 2007, MNRAS, 376,313

Hardy, A., Schreiber, M. R., Parsons, S. G., et al. 2016, MNRAS, 459, 4518

He, J. H., Szczerba, R., Hasegawa, T. I., \& Schmidt, M. R. 2014, ApJS, 210,26

Hildebrand, R. H. 1983, QJRAS, 24, 267

Hillen, M., de Vries, B. L., Menu, J., et al. 2015, A\&A, 578, A40

Hillen, M., Van Winckel, H., Menu, J., et al. 2017, A\&A, 599, A41

Holman, M. J., \& Wiegert, P. A. 1999, AJ, 117, 621

Homan, W., Danilovich, T., Decin, L., et al. 2018, A\&A, 614, A113

Jura, M. 1986, ApJ, 309, 732

Kama, M., Min, M., \& Dominik, C. 2009, A\&A, 506, 1199

Kamath, D., Wood, P. R., Van Winckel, H., \& Nie, J. D. 2016, A\&A, 586, L5

Kiss, L. L., \& Bódi, A. 2017, A\&A, 608, A99

Kluska, J., Hillen, M., Van Winckel, H., et al. 2018, A\&A, 616, A153

Kluska, J., Van Winckel, H., Hillen, M., et al. 2019, A\&A, 631, A108

Kraus, S., Kreplin, A., Young, A. K., et al. 2020, Sci, 369, 1233

Ladjal, D., Justtanont, K., Groenewegen, M. A. T., et al. 2010, A\&A, 513, A53

Lazareff, B., Berger, J. P., Kluska, J., et al. 2017, A\&A, 599, A85

Lindegren, L., Lammers, U., Hobbs, D., et al. 2012, A\&A, 538, A78

Manick, R., Kamath, D., Van Winckel, H., et al. 2019, A\&A, 628, A40

Manick, R., Van Winckel, H., Kamath, D., Hillen, M., \& Escorza, A. 2017, A\&A, 597, A129

Manick, R., Van Winckel, H., Kamath, D., Sekaran, S., \& Kolenberg, K. 2018, A\&A, 618, A21

Marconi, M., Coppola, G., Bono, G., et al. 2015, ApJ, 808, 50

Mason, K. O., Breeveld, A., Much, R., et al. 2001, A\&A, 365, L36

McMullin, J. P., Waters, B., Schiebel, D., Young, W., \& Golap, K. 2007, in ASP Conf. Ser. 376, CASA Architecture and Applications, ed. R. A. Shaw, F. Hill, \& D. J. Bell (San Francisco, CA: ASP), 127
Mohamed, S., \& Podsiadlowski, P. 2007, in ASP Conf. Ser. 372, 15th European Workshop on White Dwarfs, ed. R. Napiwotzki \& M. R. Burleigh (San Francisco, CA: ASP), 397

Monnier, J. D., \& Millan-Gabet, R. 2002, ApJ, 579, 694

Monnier, J. D., Millan-Gabet, R., Billmeier, R., et al. 2005, ApJ, 624, 832

Moschou, S.-P., Vlahakis, N., Drake, J. J., et al. 2020, ApJ, 900, 157

Nazé, Y., Broos, P. S., Oskinova, L., et al. 2011, ApJS, 194, 7

Ochsenbein, F., Bauer, P., \& Marcout, J. 2000, A\&AS, 143, 23

Oomen, G.-M., Pols, O., Van Winckel, H., \& Nelemans, G. 2020, A\&A, 642, A234

Oomen, G.-M., Van Winckel, H., Pols, O., et al. 2018, A\&A, 620, A85

Plachy, E., Bódi, A., \& Kolláth, Z. 2018, MNRAS, 481, 2986

Plachy, E., Molnár, L., Kolláth, Z., Benkő, J. M., \& Kolenberg, K. 2014, in IAU Symp. 301, Precision Asteroseismology, ed. J. A. Guzik et al. (Cambridge: Cambridge Univ. Press), 473

Pollard, K. H., \& Cottrell, P. L. 1995, in ASP Conf. Ser. 83, IAU Colloq. 155: Astrophysical Applications of Stellar Pulsation, ed. R. S. Stobie \& P. A. Whitelock (San Francisco, CA: ASP), 409

Pollard, K. R., Cottrell, P. L., Kilmartin, P. M., \& Gilmore, A. C. 1996, MNRAS, 279, 949

Pollard, K. R., McSaveney, J. A., \& Cottrelll, P. L. 2006, MmSAI, 77, 527

Primiani, R. A., Young, K. H., Young, A., et al. 2016, JAI, 5, 1641006

Prša, A., Conroy, K. E., Horvat, M., et al. 2016, ApJS, 227, 29

Sabin, L., Wade, G. A., \& Lèbre, A. 2015, MNRAS, 446, 1988

Sahai, R., Claussen, M. J., Schnee, S., Morris, M. R., \& Sánchez Contreras, C. 2011, ApJL, 739, L3

Sahai, R., Sanz-Forcada, J., Sánchez Contreras, C., \& Stute, M. 2015, ApJ, 810,77

Smith, R. K., Brickhouse, N. S., Liedahl, D. A., \& Raymond, J. C. 2001, ApJL, 556, L91

Soszyński, I., Udalski, A., Szymański, M. K., et al. 2017, AcA, 67, 297

Soszyński, I., Udalski, A., Szymański, M. K., et al. 2020, AcA, 70, 101

Stelzer, B., Micela, G., Hamaguchi, K., \& Schmitt, J. H. M. M. 2006, A\&A, 457, 223

Stelzer, B., Robrade, J., Schmitt, J. H. M. M., \& Bouvier, J. 2008, A\&A, 493, 1109

Tang, S., Grindlay, J., Los, E., \& Servillat, M. 2013, PASP, 125, 857

van Winckel, H. 2003, ARA\&A, 41, 391

van Winckel, H. 2017, in IAU Symp. 323, Planetary Nebulae: Multiwavelength Probes of Stellar and Galactic Evolution, ed. X. Liu, L. Stanghellini, \& A. Karakas (Cambridge: Cambridge Univ. Press), 231 van Winckel, H. 2018, arXiv:1809.00871

van Winckel, H., Lloyd Evans, T., Briquet, M., et al. 2009, A\&A, 505, 1221

Vega, L. D., Stassun, K. G., Montez, R., Jr., Boyd, P. T., \& Somers, G. 2017, ApJ, 839, 48

Venn, K. A., Puzia, T. H., Divell, M., et al. 2014, ApJ, 791, 98

Waters, L. B. F. M., Trams, N. R., \& Waelkens, C. 1992, A\&A, 262, L37

Watson, C. L., Henden, A. A., \& Price, A. 2006, SASS, 25, 47

Weidemann, V. 1990, ARA\&A, 28, 103

Wilms, J., Allen, A., \& McCray, R. 2000, ApJ, 542, 914 